\title{
A LARGE-AREA SEARCH FOR LOW-MASS OBJECTS IN UPPER SCORPIUS. II. AGE AND MASS DISTRIBUTIONS
}

\author{
Catherine L. Slesnick, ${ }^{1}$ Lynne A. Hillenbrand, and John M. Carpenter \\ Department of Astronomy, California Institute of Technology, Pasadena, CA 91125; \\ cslesnick@ciw.edu, lah@astro.caltech.edu,jmc@astro.caltech.edu \\ Received 2008 April 21; accepted 2008 August 4
}

\begin{abstract}
We present continued results from a wide-field, $\sim 150 \mathrm{deg}^{2}$, optical photometric and spectroscopic survey of the northern part of the $\sim 5$ Myr old Upper Scorpius OB association. Photometry and spectral types were used to derive effective temperatures and luminosities and place newly identified association members onto a theoretical Hertzsprung-Russell diagram. From our survey, we have discovered 145 new low-mass members of the association and determined $\sim 10 \%$ of these objects to be actively accreting material from a surrounding circumstellar disk. Based on comparison of the spatial distributions of low- and high-mass association members, we find no evidence for spatial segregation by mass within the northern portion of the association. Measured data are combined with pre-mainsequence evolutionary models to derive a mass and age for each star. Using Monte Carlo simulations, we show that, taking into account known observational uncertainties, the observed age dispersion for the low-mass population in USco is consistent with all stars forming in a single burst $\sim 5 \mathrm{Myr}$ ago, and we place an upper limit of $\pm 3 \mathrm{Myr}$ on the age spread if the star formation rate has been constant in time. We derive the first spectroscopic mass function for USco that extends into the substellar regime and compare these results to those for three other young clusters and associations.
\end{abstract}

Subject headings: open clusters and associations: individual (Upper Scorpius) — stars: fundamental parameters stars: low-mass, brown dwarfs - stars: luminosity function, mass function -

stars: pre-main-sequence

\section{INTRODUCTION}

The review of Shu et al. (1987) laid the basic framework for how isolated stars are created. However, most stars do not form in isolation but rather in groups (i.e., clusters or associations; Gomez et al. 1993; Lada \& Lada 1995; Carpenter 2000), and many details of the processes involved in stellar group formation remain unexplained. In particular, do all members of an individual group form in a single burst, or is star formation a lengthy process? The distribution of stellar masses formed within a stellar group is known as the initial mass function (IMF). Is the IMF universal, or does it vary with either star formation environment or time?

The phenomenon of rapid (1-2 Myr) clustered star formation has been found in almost all nearby young associations (e.g., Lada $\&$ Lada 2003). The large numbers of very young stars and apparent lack of more evolved (5-10 Myr old) objects in star-forming regions contrast with ages of a few tens of megayears (e.g., Blitz \& Shu 1980) inferred for molecular clouds. Either star formation takes place for only a small fraction of the cloud lifetime, molecular clouds themselves live only a few megayears (e.g., Hartmann et al. 2001), or older group members have been missed in observational surveys that are often biased toward stars that are still actively accreting or that possess an optically thick disk. Observational surveys capable of detecting the entire age range present within a stellar group are needed if we are to begin to understand the time dependence of star formation within a molecular cloud.

The basic structure of the field star IMF in the mass range $\sim 0.5-10 M_{\odot}$ is well characterized by a power law with slope $d N / d M \propto M^{-2.35}$, as derived originally by Salpeter (1955). However, while the shape of the stellar mass function between $\sim 0.5$

\footnotetext{
${ }^{1}$ Current address: Department of Terrestrial Magnetism, Carnegie Institute of Washington, Washington, DC 20015.
}

and $10 M_{\odot}$ is near universal, below $\sim 0.5 M_{\odot}$ the shape of the mass function may be strongly dependent on environment within the parental molecular cloud (Luhman et al. 2003b; Slesnick et al. 2004). Young stellar clusters and associations are particularly valuable for examining the shape of the low-mass IMF because contracting low-mass pre-main-sequence (PMS) stars and brown dwarfs are $2-3.5$ orders of magnitude more luminous than their counterparts on the main sequence. Thus, despite the fact that they are farther away, young stars and brown dwarfs can be more readily detected than stars of equivalent mass in the field.

The Upper Scorpius OB association (USco) is the closest (145 pc; de Zeeuw et al. 1999) young OB association to the Sun, with 120 members more massive than $\sim 1 M_{\odot}$ and an associated population of $\sim 400$ known low-mass objects (e.g., Preibisch et al. 1998). Because of its proximity and large population that spans the range from $<0.02$ to $>10 M_{\odot}$, USco is an ideal region in which to study the mass distribution of stars in $\mathrm{OB}$ associations. Furthermore, at an age of $\sim 5 \mathrm{Myr}$ (Preibisch et al. 2002), USco is young enough that we may be able to measure the ages of member stars to a precision of less than a million years, and old enough that an ongoing episode of star formation lasting several millions of years could be detectable. A major difficulty faced by studies of the USco region is that the high-mass members alone span $>300 \mathrm{deg}^{2}$ on the sky. Obtaining a complete census of the association's low-mass population is thus a formidable task as one must identify faint objects over a very large spatial region. While there exist several techniques to identify young stars, many observational signatures (e.g., $\mathrm{H} \alpha$ emission or near-infrared excess) probe accretion processes and circumstellar material rather than characteristics intrinsic to the stars themselves.

Recently, deep, multicolor imaging surveys combined with spectroscopic follow-up have proven successful in identifying low-mass stars and brown dwarfs without active accretion, as well 
as classical T Tauri-type objects. Young PMS objects still undergoing contraction are cooler (i.e., redder in color) and/or more luminous (i.e., brighter in magnitude) than their main-sequence counterparts. In nearby regions, candidate PMS stars can be identified through photometric colors and magnitudes that are systematically different from those of the bulk field star population. Spectroscopic follow-up observations allow assessment of surface gravity diagnostics that can be used to distinguish bona fide young PMS stars from foreground field dwarfs and reddened background giants. Previous imaging and spectroscopic surveys in USco include studies by Preibisch et al. (2001, 2002), whose work yielded 166 new low-mass PMS objects. Lodieu et al. (2006, 2008), Martín et al. (2004), and Ardila et al. (2000) together identified 90 members of USco with spectral type M6 or later. Thus far, over 400 low-mass $\left(M<0.6 M_{\odot}\right)$ members have been identified in USco through X-rays, $\mathrm{H} \alpha$ emission, photometry, and/or spectroscopy. However, most searches have been limited to small subregions (smaller than a few square degrees) or bright objects ( $R \lesssim 16 \mathrm{mag}$ ). Given the derived mass function and number of observed high-mass stars in USco, and assuming that the highand low-mass objects share the same spatial distribution, Preibisch et al. (2002) estimate that the entire USco region should contain $>1500$ young, low-mass objects with $M<0.6 M_{\odot}$, most of which are yet to be discovered.

In Slesnick et al. (2006a, hereafter Paper I) we introduced our wide-field photometric survey covering $\sim 150 \mathrm{deg}^{2}$ of USco. In that work, we presented optical spectroscopic observations of 62 photometrically selected candidate PMS stars, of which 43 were confirmed by us to be new USco members. In this companion paper we present spectra for an additional 178 candidates observed at either Palomar Observatory or Cerro Tololo InterAmerican Observatory (CTIO). In $\S 2$ we give a brief overview of the photometric and spectroscopic surveys and present new USco members discovered as a result of recent observations. For the remainder of the paper beyond $\S 2$, we discuss results for new members presented here together with those for association members identified in Paper I. In $\S 3$ we discuss $\mathrm{H} \alpha$ emission profiles and accretion signatures in USco. In $\S 4$ we compare the spatial distribution of the high- and low-mass populations. Finally, in $\S 5$ we construct a Hertzsprung-Russell (H-R) diagram and present a discussion of the age and mass distributions derived for the members of USco presented in our work, as well as a comparison of distributions derived for other nearby star-forming regions.

\section{OBSERVATIONS}

\subsection{Quest-2 Photometric Observations and Candidate Member Selection}

Drift-scan observations were taken with the Quest-2 camera (Rabinowitz et al. 2003; Baltay et al. 2007) on the 48 inch (1.2 m) Samuel Oschin Schmidt Telescope at Palomar Observatory. Details of the observations and data analysis are given in Paper I, Slesnick et al. (2006b, hereafter SCH06), and Slesnick (2007). Three $4.6^{\circ}$ wide scans, centered at $\delta=-15.7^{\circ},-19.5^{\circ}$, and $-23.3^{\circ}$, were each observed between R.A. of $15^{\mathrm{h}} 46^{\mathrm{m}}$ and $16^{\mathrm{h}} 36^{\mathrm{m}}$ for a total survey area of $\sim 150 \mathrm{deg}^{2}$. The scan centered at $\delta=$ $-19.5^{\circ}$ was observed $3-4$ times per night on seven consecutive, photometric nights between 2004 June 20 and 26. The other two scans were observed once during this period.

$B, R, I$ photometric data were calibrated to a system closely aligned with Sloan $g, r, i$ magnitudes as described in SCH06 and Slesnick (2007). Magnitudes for all the new USco members identified in this study (based on a combination of photometry and spectroscopy; see $\S 2.4$ ), both those newly identified in this work and those identified originally in Paper I, are given in Table 1. Our goal is to use the photometry to isolate PMS stars from the field star population that dominates the $\sim 2$ million member source catalog. As discussed in $\S 1$, nearby young stars occupy a sequence in an optical color-magnitude diagram (CMD) that is systematically brighter and/or redder than the sequence occupied by most field stars and thus can be identified based on their colors and magnitudes. While the data have been calibrated to approximate Sloan magnitudes (for more details see Slesnick 2007), they are not precisely on any standard system, and we did not translate theoretical isochrones into the Quest-2 system. Thus, we did not select candidates based on isochronal data and instead considered as candidate PMS stars all sources redward of a linear approximation of the $1 \%$ data contour in an $r, r-i$ CMD (Fig. 1). This criterion roughly corresponds to selecting all candidates redward of a $30 \mathrm{Myr}$ isochrone.

In addition to optical colors, the infrared colors and magnitudes of potential PMS candidates were also considered. We matched the entire source catalog to the Two Micron All Sky Survey (2MASS; Skrutskie et al. 2006) and excluded from further consideration sources that did not have a $2 \mathrm{MASS}$ counterpart. This criterion served to remove artifacts detected in the two drift scans for which we did not have repeated observations. However, as discussed in Paper I and SCH06, requiring a 2MASS detection biases the list of potential PMS candidates against faint blue sources. The position of each star on a near-infrared color-color diagram was examined, and any star with $J-H, H-K_{s}$ colors consistent with those of background giants $\left[(J-H)>0.6\left(H-K_{S}\right)+0.6\right.$ or $\left.(J-H)>1.69\left(H-K_{s}\right)+0.29\right)$; Bessell \& Brett 1988] was excluded. We additionally considered $r-K_{s}$ colors and adopted the selection criterion $r<2.57\left(r-K_{s}-3\right)+12.8$ as outlined in Paper I. After all selection criteria were applied, the final candidate list contained $\sim 1700$ candidate low-mass PMS stars identified from $\sim 2$ million sources detected in the Quest-2 survey. Of these stars, 90 correspond to previously known low-mass members of USco (Ardila et al. 2000; Preibisch et al. 2002; Martín et al. 2004). The candidates were spread out over the entire $\sim 150 \mathrm{deg}^{2}$ survey area, which encompasses $\sim 40 \%$ of the total spatial area spanned by the high-mass members. Due to interstellar extinction and distance effects, optical and near-infrared colors and magnitudes alone are not a unique indicator of youth. Therefore, it is necessary to obtain spectroscopic follow-up observations for each photometrically selected candidate. These data allow for spectral type determination and can confirm the presence of spectral features indicative of youth.

\subsection{Palomar Spectroscopy}

Moderate-resolution spectra of 105 PMS star candidates were taken with the Double Spectrograph on the Palomar 200 inch $(5.08 \mathrm{~m})$ telescope during the nights of 2006 May 16-19 and June 1-2. All data were taken with the red side of the spectrograph through either the $1.5^{\prime \prime}$ or the $2^{\prime \prime}$ slit using a $5500 \AA$ dichroic and a 316 line $\mathrm{mm}^{-1}$ grating blazed at $7500 \AA$. This setup produced wavelength coverage from 6300 to $8825 \AA$ at a resolution of $R \sim 1250$. Typical exposure times were 300-900 s, and up to $1800 \mathrm{~s}$ for the faintest targets $(r \sim 20)$. Spectrophotometric standard stars (Massey et al. 1988) were observed throughout each night for flux calibration. All sources were processed, extracted, and calibrated using standard IRAF tasks.

Spectral analyses for these observations were carried out as detailed in Paper I, SCH06, and Slesnick (2007). Both spectral type (temperature) and surface gravity (age) determinations were first made through quantitative measurements of the TiO-7140, TiO-8465, and Na-8190 indices (defined in Paper I), which 


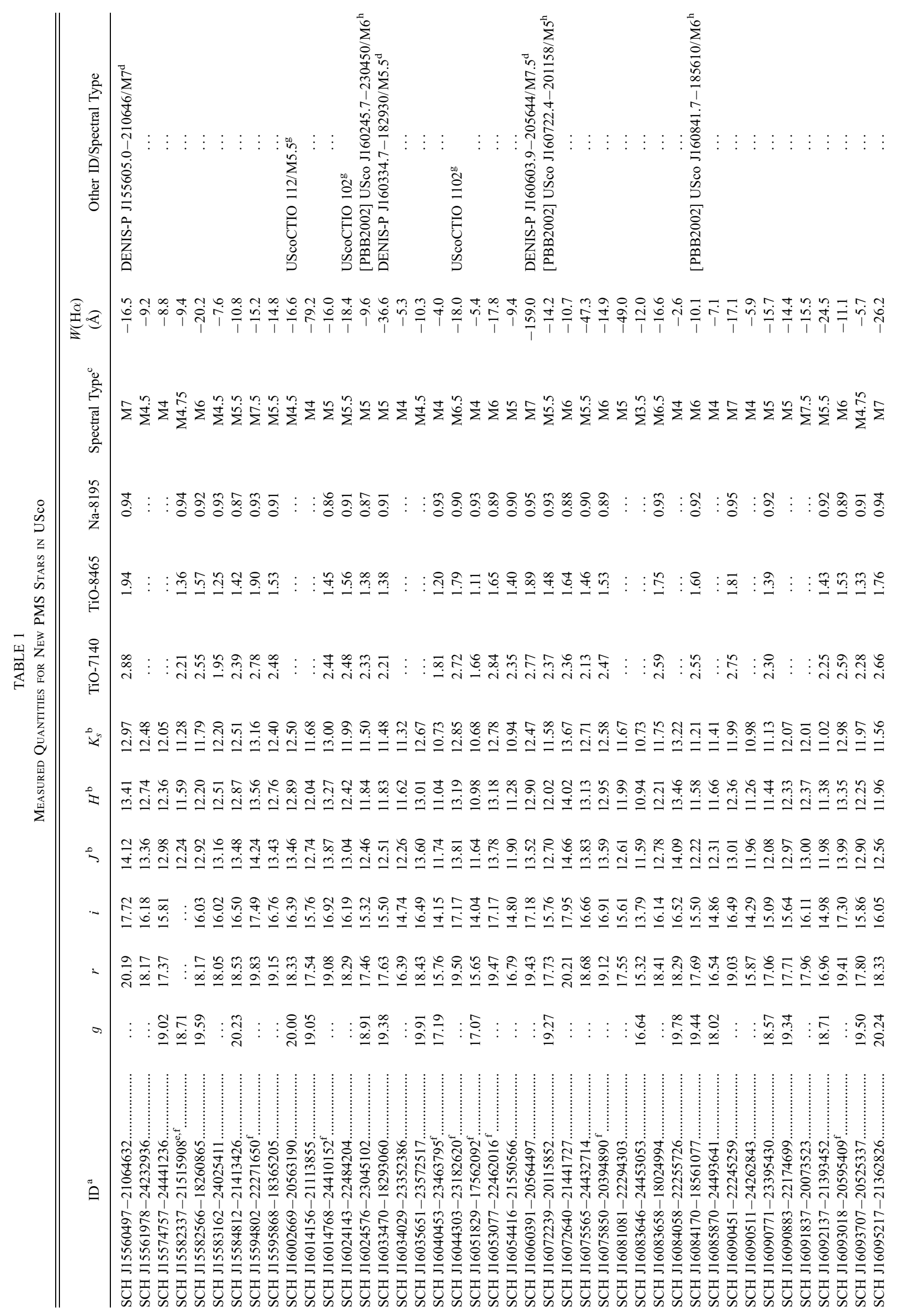




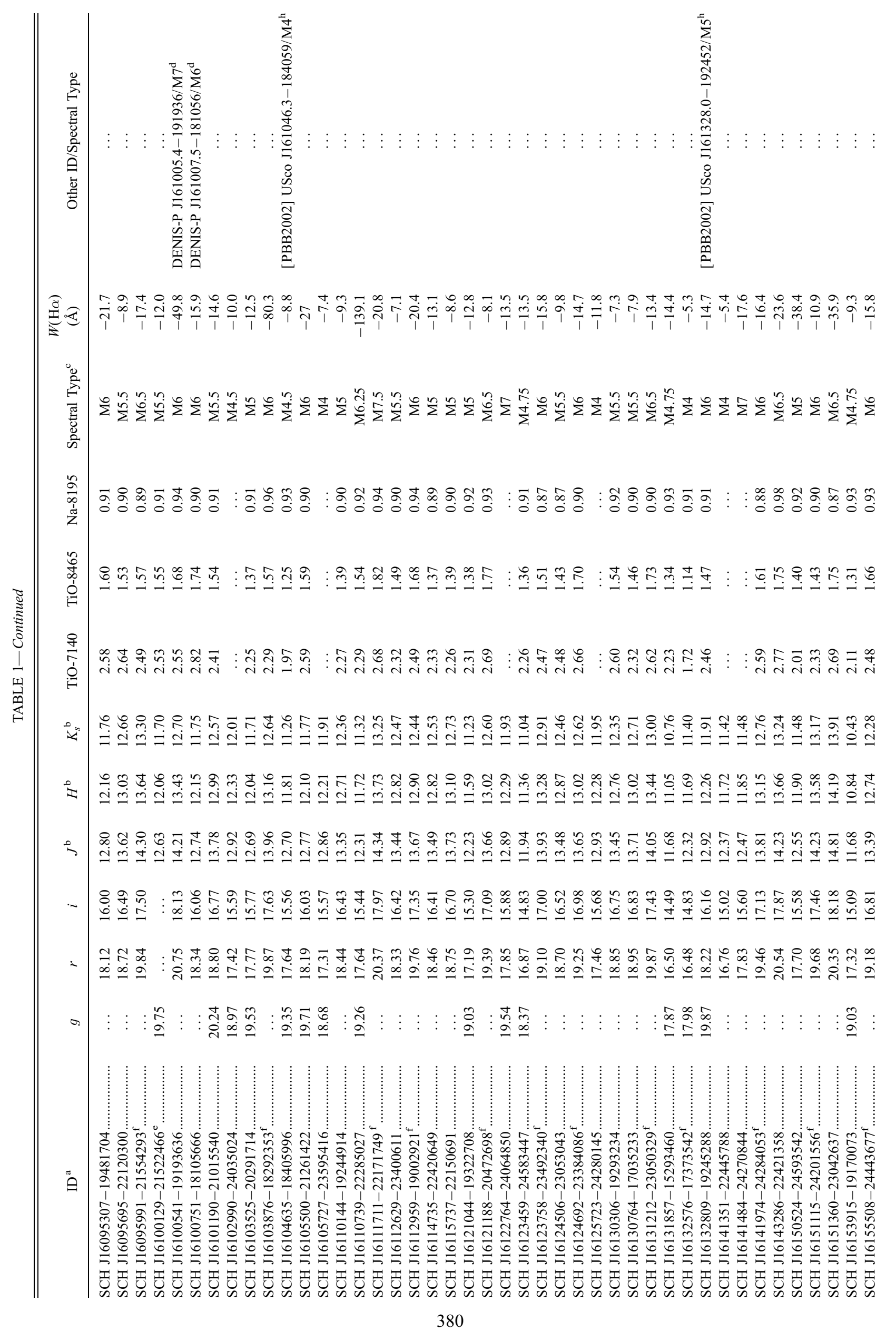




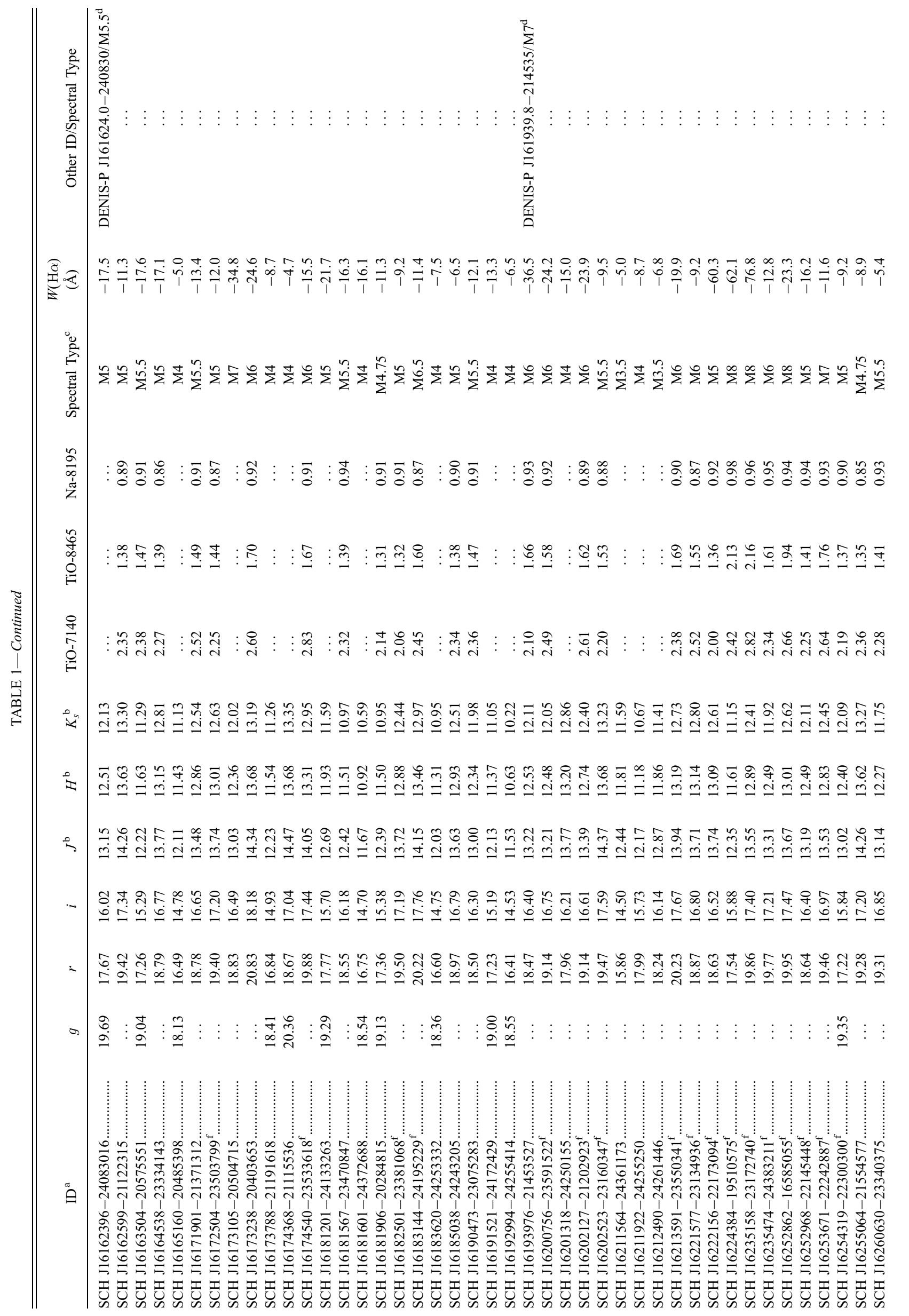




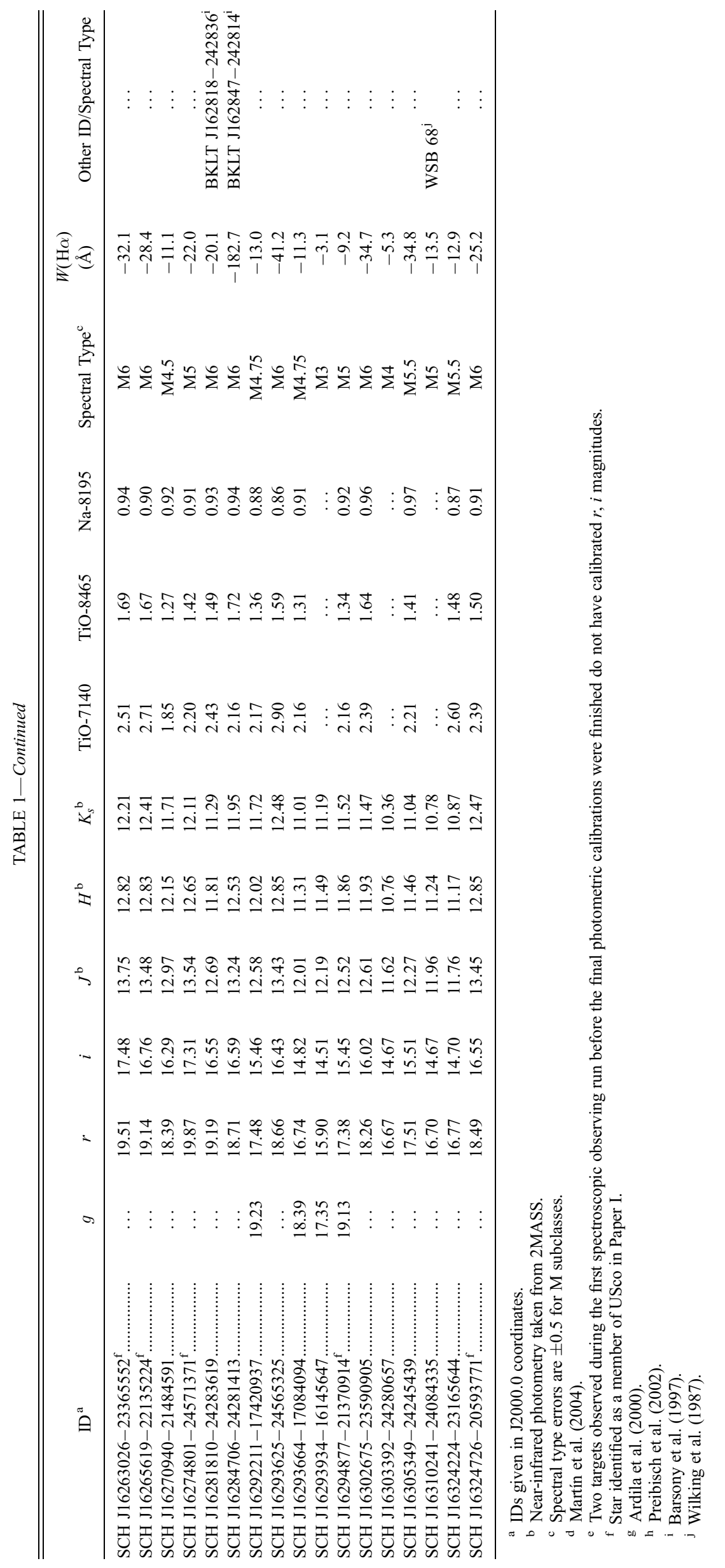




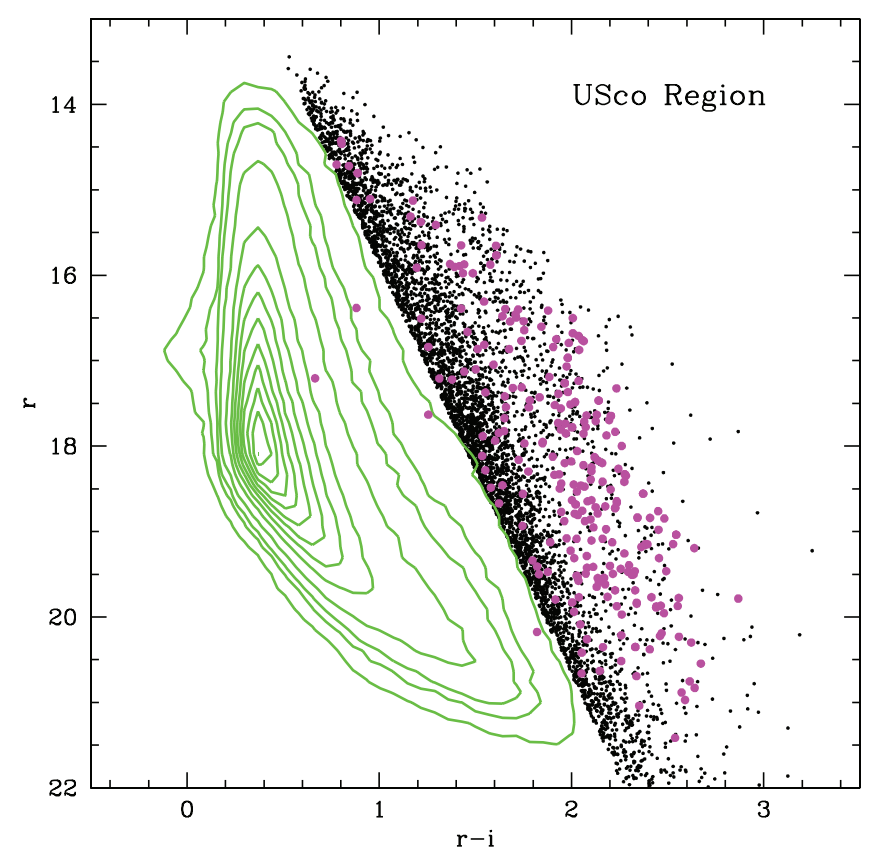

FIG. 1.-Optical CMD of all Quest-2 sources in the final USco catalog with

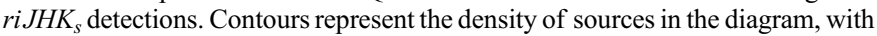
contour levels at $1 \%, 2 \%, 5 \%$, and $10 \%-90 \%$ of the peak value. Objects redward of a linear approximation of the $1 \%$ contour are shown as discrete points. Objects for which we have spectral data are shown as large symbols. Photometry for four of the targets has changed significantly since the first spectroscopic observations were taken (see SCH06) such that they would no longer be considered candidates.

measure the strength of molecular and atomic absorption features present at optical wavelengths in the spectra of K- and M-type stars. Figure 2 shows spectral indices for 167 PMS spectral candidates in the USco region observed at Palomar (105 presented here for the first time and 62 presented in Paper I). The left panel shows measurements of temperature-sensitive indices used to aid in spectral type determination. We find that 14 outliers sit below the main locus of data points. In all cases, the star is confirmed to exhibit low gravity signatures (see below), and we attribute the position in Figure 2 to a small amount of veiling or reddening. A detailed explanation of the possible effects of these processes on the classification indices is given in Paper I and SCH06.

The surface gravity-sensitive Na-8190 index (Fig. 2, right panel) allows us to easily separate the bona fide low-gravity (i.e., young) objects from contaminant dwarf stars over the spectral type range $\sim \mathrm{M} 3-\mathrm{M} 8$. We find that a large fraction $(\sim 65 \%)$ of the candidate objects have measured Na-8190 indices consistent with their having surface gravity less than that of field dwarfs at similar spectral types. The $\mathrm{TiO}$ and $\mathrm{Na}$ quantitative spectral indices were used to aid in classification only. All final spectral type and gravity determination was done by visually comparing each spectrum to a grid of spectral standard stars observed throughout the observing run.

\subsection{Cerro Tololo Spectroscopy}

Additional spectra of PMS star candidates in USco were taken at CTIO using the Hydra multifiber spectrograph on the Blanco $4 \mathrm{~m}$ telescope during the nights of 2005 July 24-28. In total, 26 pointings were observed with the Site $2 \mathrm{k} \times 4 \mathrm{k}$ CCD through the KPGLF grating. The setup provided wavelength coverage from $\sim 6300$ to $8660 \AA$ at a resolution of $1.15 \AA \mathrm{pixel}^{-1}$.

Fibers were placed preferentially on stars meeting the criteria outlined in $\S 2.1$, thus providing a sample of candidates analogous to those observed at Palomar. Remaining fibers were placed on stars meeting one or more of the criteria outlined in $\S 2.1$. For the practical requirements of the Hydra observations, stars were assigned to either "bright" pointings (corresponding to $r \lesssim 16.5$ ) or "faint" pointings (corresponding to $16.5 \lesssim r \lesssim 18.5$ ). Exposure times ranged from 900 to $1800 \mathrm{~s}$ for bright pointings (dependent on weather conditions) and were set at $2700 \mathrm{~s}$ for faint
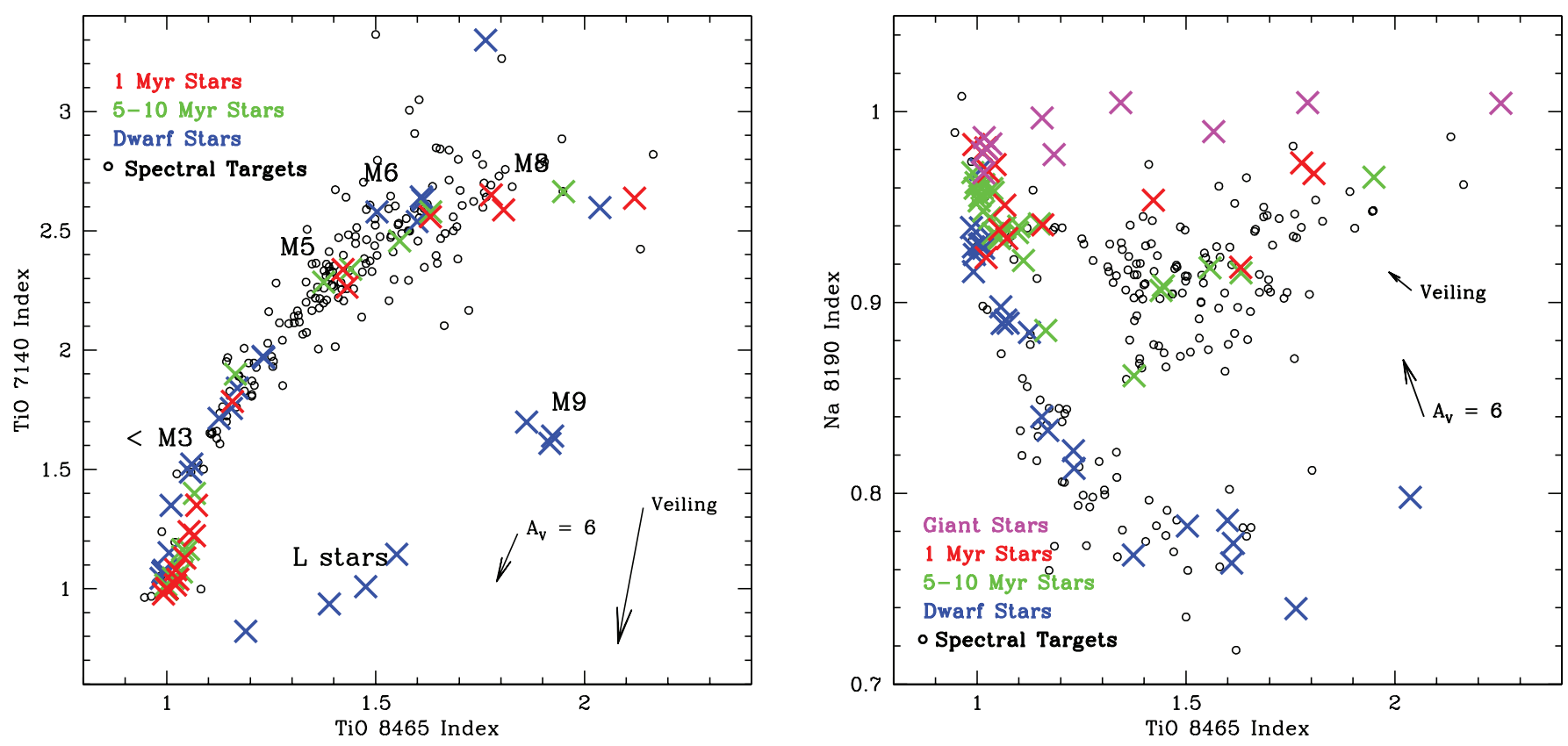

FIg. 2.-Left: Temperature-sensitive TiO-7140 vs. TiO-8465 indices. Right: TiO-8465 index vs. gravity-sensitive Na-8190 index. In both panels, blue crosses represent measured indices for a sample of old stars comprised of field dwarfs and members of the Hyades ( $\sim 650 \mathrm{Myr})$, Pleiades ( $\sim 115 \mathrm{Myr})$, and AB Dor ( 75-150 Myr) associations. Green crosses show measured indices for intermediate-age spectral standards from $\beta$ Pic ( $\sim 11 \mathrm{Myr})$, TW Hya ( $\sim 8 \mathrm{Myr})$, and Upper Sco ( $\sim 5 \mathrm{Myr})$. Red crosses show measured indices for young Taurus members ( $\sim 1-2 \mathrm{Myr})$. Magenta crosses in the right panel represent measured indices for giant standard stars. In both panels, black circles are measured indices for USco PMS candidates observed at Palomar. The effects of extinction and veiling are shown as vectors (see Paper I; SCH06). 
pointings. In total, 10 bright and 16 faint pointings were observed during the five nights. At each fiber configuration, we observed spectra of a comparison lamp and a quartz lamp to allow determination of the dispersion solution and throughput correction during data reduction. Biases and dome flats were taken each afternoon with all working fibers put in the "large circle" configuration. Milk flats (see below) were taken once during the observing run.

\subsubsection{Image Processing and Sky Subtraction}

All frames were first preprocessed (bias correction and trimming) in IRAF using the ccdproc task. Milk flats were obtained on the second afternoon of observing. This type of observation is an exposure of the daytime sky and is taken through a plate of milky glass placed between the output of the fibers and the spectrograph camera. The purpose of milk flats is to allow removal of CCD pixel-to-pixel variations from the data in the absence of fibers. After median-combining all observed milk flats, a spectral response image was created by smoothing in both $X$ - and $Y$-directions. The original combined milk flat was divided by the spectral response image to create an image with a value of 1.0 everywhere except where pixel-to-pixel variations exist. All data and calibration frames were divided by this image.

Subsequent data reduction was done using the IRAF dohydra task. All data for a given night were divided by the dome flat taken that afternoon. Individual fiber-to-fiber throughput corrections (which change for each configuration) were made from division of each object frame by the corresponding quartz lamp spectra taken in the same fiber configuration. Wavelength calibration was carried out by matching each object spectrum to the corresponding lamp spectrum observed through the same fiber.

For each pointing, a single sky spectrum was made using the sky generating tasks within dohydra. Typically, $\sim 5-20$ fibers were placed on sky during each observation. The dohydra sky generating task first allows rejection of any anomalous sky spectra that may have inadvertently fallen on a star or a clump of nebulosity. Remaining sky spectra are averaged together using a $3 \sigma$ clipping algorithm. The Hydra field of view is $40^{\prime}$ on a side. We found that subtraction of a single sky spectrum for all spectra within a field often produced insufficient sky subtraction in that, for a given spectrum, all sky lines could be systematically overor undersubtracted. To correct this problem, for each spectrum we computed the flux in a single sky line chosen to be toward the center of the spectrum $(\sim 7316 \AA)$ and to be sufficiently isolated that it was not blended with any other sky or stellar lines. Object spectra were scaled such that the flux computed in the central sky line matched that of the sky spectrum and the sky spectrum was subsequently subtracted.

\subsubsection{Classification}

Spectral observations taken through fibers produce flux that varies as a function of wavelength, dependent on the fiber configuration (i.e., how the relevant fiber is bent and stretched to place it into position on the star). Thus, flux correction of fiber data is inherently difficult because it is not practical to observe a calibrator star through every fiber at every configuration. We were therefore not able to flux-correct the Hydra spectra in a manner analogous to the Palomar data and could not use the quantitative spectral indices (which rely on flux-corrected spectra) to aid in classification. Although the overall spectral shape is not meaningful because the spectra are not flux corrected, the depth of absorption and emission features is not substantially affected. During observations, we observed a range of known dwarf and giant stars (spectral types K0-M8). In addition, we observed known intermediate-age PMS members of the $\sim 30$ Myr old Tucanae
Hor association (Mamajek et al. 2004) with early M spectral types. Spectra of previously identified K0-M8-type USco members were observed in pointings toward USco along with program candidates. All spectra of candidate PMS stars were classified by hand through comparison to each other and to standard stars observed with Hydra.

In total, the Hydra observations yielded $\sim 1150$ spectra (of varying quality). We first classified all of the spectra into broad categories. Approximately 450 were determined to be mid-K to $\mathrm{M}$ "late-type" stars based on the presence of TiO molecular absorption in their spectra and a lack of $\mathrm{H} \alpha$ absorption. The largest constituent of the spectral sample ( 650 stars) were "midtype" stars with spectral types late F through early K. These stars are characterized by a lack of $\mathrm{TiO}$ molecular absorption but the presence of $\mathrm{Ca}$ II triplet $(\lambda \lambda 8498,8542,8662)$ and $\mathrm{H} \alpha$ atomic absorption. Absorption from the Ba II, Fe I, Ca I blend at $6497 \AA$ begins to appear at late F/early $\mathrm{G}$ types and increases in strength compared to $\mathrm{H} \alpha$ absorption through $\mathrm{K}$ spectral types. Thirty of the late $\mathrm{F}$ to early K-type stars have $\mathrm{H} \alpha$ emission present in their spectra. The remaining 50 stars observed with Hydra were determined to have spectral types A-F with deep $\mathrm{H} \alpha$ and Ca II triplet absorption but noticeably lacking any absorption at the $\lambda 6497$ blend. As expected, we saw no evidence of He absorption in any of the spectra and conclude that our survey did not probe B- or O-type stars.

Magnitude histograms for the samples of observed midtype and late-type stars are shown at $r$ and $J$ band (Fig. 3), along with the range of magnitudes expected for members of USco at these spectral types. We have assumed for this calculation that all members of USco are 5 Myr old, $145 \mathrm{pc}$ away, and have $0 \leq A_{V} \leq 2$ (Paper I). As can been seen, most of the stars classified as midtype do not appear to be members of USco based on their observed magnitudes and spectral types. This sample is instead likely dominated by reddened field dwarfs and background giants. This result is not surprising, considering that most of these stars were observed through fibers placed on stars in the field of view that did not meet all three criteria for candidate selection (see $\S \S 2.3$ and 2.1) and were not part of the $\sim 1700$ member candidate list.

Thus far, we have derived detailed (at the $\sim 0.5$ subclass level) classifications from these data only for those stars whose colors and magnitudes meet all of the selection criteria outlined in Paper I and SCH06, thus providing a sample selected in exactly the same manner as the stars observed at Palomar. This sample was determined to consist entirely of late K- and M-type stars, $\sim 50 \%$ of which have low surface gravity consistent with association membership. We present here results for these stars, together with results from the Palomar spectral observations.

\subsection{Summary of Observations}

We identified a total of $\sim 2$ million sources in the $\sim 150 \mathrm{deg}^{2}$ area covered by the Quest-2 imaging survey of the northern part of USco. The survey encompassed 56 out of 120 high-mass association members identified with Hipparcos (de Zeeuw et al. 1999 ) and $\sim 40 \%$ of the total spatial area spanned by the highmass members. Using a combination of Quest-2 $g, r, i$ and 2MASS $J, H, K_{s}$ magnitudes, we selected $\sim 1700$ candidate young stars based on placement in color-color and color-magnitude diagrams. Specifically, to be considered as a candidate PMS star, the object was required to be within the reddest $1 \%$ (in $r-i$ color) of stars observed in our survey and to have infrared colors inconsistent with field giants.

We obtained optical spectra for $\sim 15 \%$ of the photometric candidates selected to be among the reddest of the photometric candidates across all magnitudes $(14 \lesssim r \lesssim 20)$ probed. The goal of 


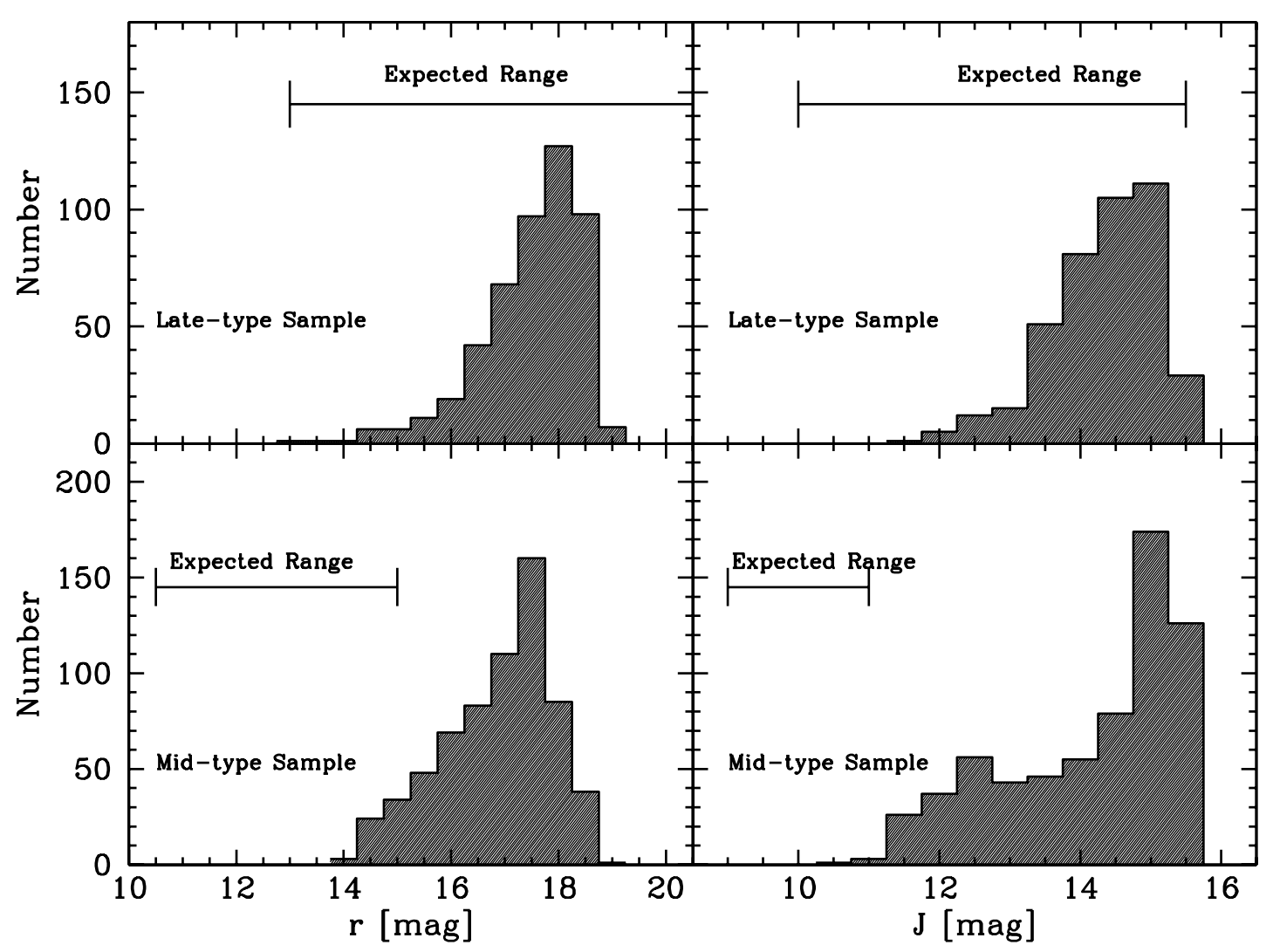

FIG. 3.- Histograms of $r$-and $J$-band magnitudes for targets of the Hydra spectral observations. The top histograms show data for stars classified as spectral type late K

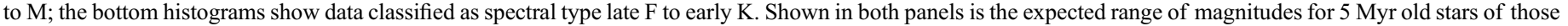
spectral types at the distance of USco.

the spectroscopic observations is to measure spectral type and confirm low gravity signatures consistent with bona fide PMS stars for each photometric candidate. From the 105 Palomar spectral observations presented here $(\S 2.2)$, we identified 66 new USco members with spectral types ranging from M3 to M8. We present an additional 36 new members with spectral types M3-M8 identified from CTIO observations. Quest-2 and 2MASS magnitudes for photometric candidates determined spectroscopically to be field dwarfs are given in Table 3 (see the Appendix).

For the remainder of this paper we discuss together results from both the 102 new low-mass USco members presented here and the 43 members presented in Paper I. Magnitudes and spectral measurements (i.e., spectral type, $\mathrm{H} \alpha$ equivalent width, and spectral indices, when applicable) for the 145 members identified by us are given in Table 1. Together, these stars represent a uniformly selected sample of new low-mass USco members that is free from bias in regards to circumstellar material and activity.

\section{EMISSION-LINE OBJECTS}

The most prominent emission line observed in the spectra of new members is $\mathrm{H} \alpha$, which, seen in the spectra of young stars and brown dwarfs, is created via one of several mechanisms. Weak, narrow $\mathrm{H} \alpha$ lines are presumed to originate from active chromospheres, whereas strong, broad, and/or asymmetric lines can be produced from high-velocity, infalling accretion or strong winds. Barrado y Navascués \& Martín (2003) have proposed an empirical, spectral type- $\mathrm{H} \alpha$ equivalent width $[W(\mathrm{H} \alpha)]$ relation to describe the upper limit of nonaccreting stars and brown dwarfs based on the chromospheric saturation limit observed in the Pleiades, $\alpha$ Per, and IC 2391 open clusters. These clusters are sufficiently old (50-125 Myr) that accretion should not be present, and any observed $\mathrm{H} \alpha$ emission is assumed to be produced entirely from chromospheric activity. Figure 4 plots measured $\mathrm{H} \alpha$ equivalent widths as a function of spectral type for the 145 members of USco presented here, shown with the Barrado y Navascués \& Martín (2003) empirical accretor/nonaccretor division. Notably, every new member identified in our work shows $\mathrm{H} \alpha$ in emission. Many stars and brown dwarfs exhibit very strong $\mathrm{H} \alpha$ emission (see also Table 1) at levels substantially above the accretor/ nonaccretor division and thus are possibly still undergoing active accretion.

We determined an empirical criterion for identifying objects with $\mathrm{H} \alpha$ excess emission based on our data. At an age of $\sim 5 \mathrm{Myr}$, we assume that the bulk of our sample is no longer accreting and compute median values of $\mathrm{H} \alpha$ emission as a function of spectral type using $1 \sigma$ clipping to remove any bias from outliers. For most bins, we define a star to have an $\mathrm{H} \alpha$ excess if it exhibits emission at a level greater than $3 \sigma$ above the median value for its spectral type, where $\sigma$ is the dispersion about the median for stars at a given spectral type. Stars with such strong $\mathrm{H} \alpha$ emission are likely to be accreting and are assumed such for the remainder of this work. These sources are distinguished in Figure 4. For spectral type bins earlier than M4 and later than M7, we have identified fewer than five stars per spectral type. Thus, for these bins we do not have enough measurements to derive a statistically representative value for median $\mathrm{H} \alpha$ emission and we do not consider any stars in these bins to be in our sample of $\mathrm{H} \alpha$ excess, accreting sources. However, we note that two M8 stars (including the possible binary discussed in Paper I, $\S$ 4.2) sit above the Barrado y Navascués \& Martín (2003) accretor/nonaccretor dividing line. 


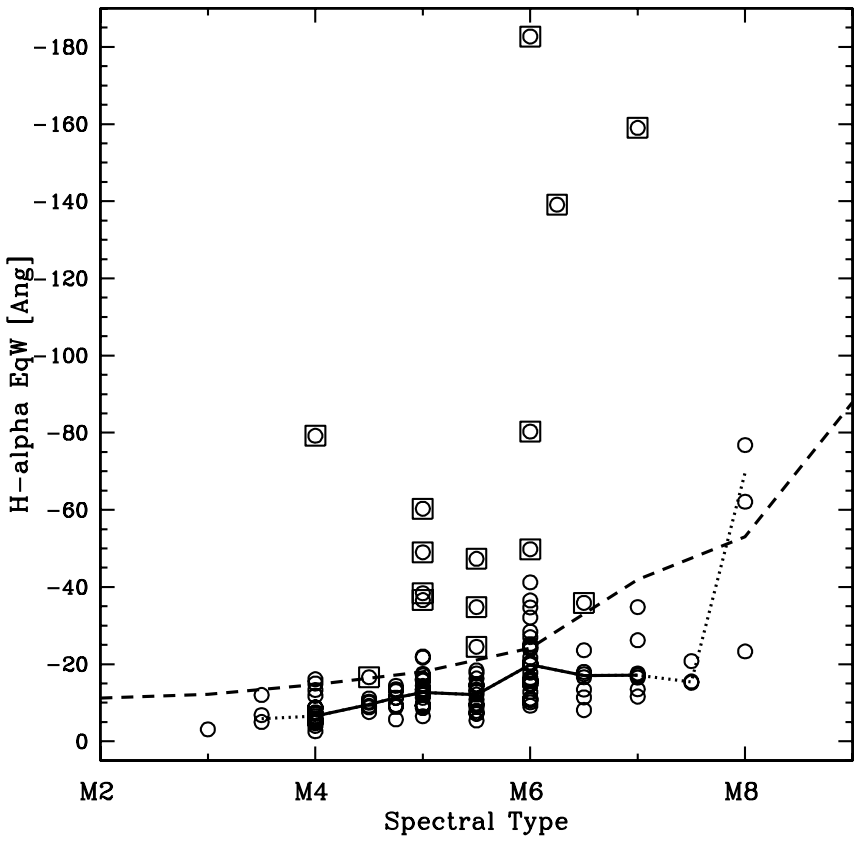

FIG. 4.-Measured $\mathrm{H} \alpha$ equivalent widths for all 145 low-mass USco members presented here, shown as a function of spectral type. The dashed line represents the empirical accretor/nonaccretor upper limit derived by Barrado y Navascués \& Martín (2003). The solid line represents the median $W(\mathrm{H} \alpha)$ for each spectral type. The spectral type bins earlier than M4 and later than M7 do not have enough measurements to derive a statistically representative value for median $\mathrm{H} \alpha$ emission, and we do not consider any star outside the M4-M7 spectral type range to be in our sample of $\mathrm{H} \alpha$ excess sources. Boxed objects have $\mathrm{H} \alpha$ emission in excess of $3 \sigma$ above the median value for their spectral type and are considered by us to be actively accreting.
Based on the above criterion, we find 15 objects that exhibit $\mathrm{H} \alpha$ emission with sufficient strength to be considered by us to be actively accreting. Spectra for accreting stars and brown dwarfs are shown in Figure 5. In addition to $\mathrm{H} \alpha$ emission, many of these stars (SCH J16014156-21113855, SCH J16222156-22173094, SCH J16150524-24593542, SCH J16033470-18293060, SCH J16075565-24432714, SCH J16284706-24281413, SCH J16103876-18292353, SCH J16110739-22285027, SCH J16060391-20564497) also exhibit He I (26678) emission, which is commonly seen in spectra of classical T Tauri-type objects. Two accreting sources lie very close (within $\sim 1^{\circ}$ ) to the young ( $\leqslant 1 \mathrm{Myr}) \rho$ Ophiuchi molecular cloud ( $\rho$ Oph; $\alpha=$ $16^{\mathrm{h}} 25^{\mathrm{m}} 35.118^{\mathrm{s}}, \delta=-23^{\circ} 26^{\prime} 49.84^{\prime \prime}$, J2000.0). However, because $\rho$ Oph lies slightly in front of USco (Loinard et al. 2008), if these stars were dynamically ejected $\rho$ Oph members, we would expect to see them exhibit systematically higher luminosities than USco members of similar spectral type. Based on the derived H-R diagram from these data (see $\S 5.1$ ), this phenomenon is not observed, and we include these two stars in our sample of accreting members of USco. We find that at an age of $\sim 5 \mathrm{Myr}$ (see $\S 5.2$ ), $15 / 145$ (or $\sim 10_{-3}^{+3} \%$ ) of low-mass association members (spectral type $\leq \mathrm{M} 7$ and $\geq \mathrm{M} 4$ ) are observed to be accreting based on the strength of $\mathrm{H} \alpha$ emission present in their spectra. If we were to use the Barrado y Navascués \& Martín (2003) accretion boundary instead of our own empirical classification, we would have determined that 23/145 (16\%) low-mass stars and brown dwarfs in our sample are still accreting. In comparison, Guieu et al. (2006) find $\sim 65 \%$ (20/31) of 1 Myr old low-mass objects in the subclusters of Taurus to be actively accreting based on the strength of $\mathrm{H} \alpha$ emission observed in their spectra compared to the Barrado y Navascués \& Martín (2003) accretion boundary. Thus, a significant fraction of very low mass stars and brown dwarfs must stop

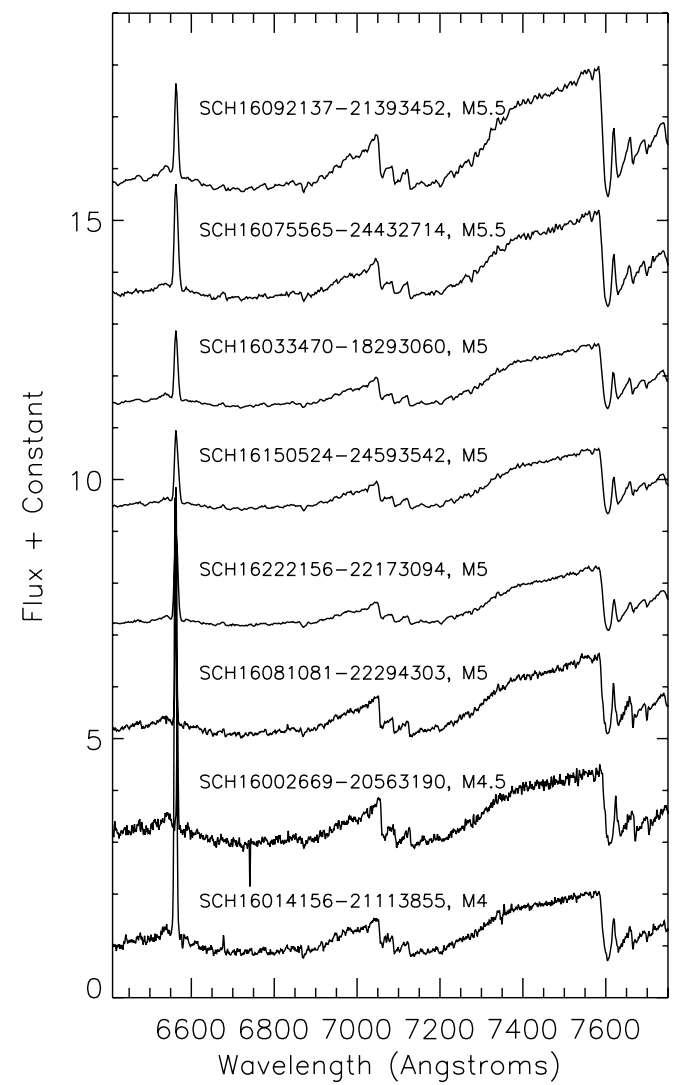

FIG. 5.- Spectra of the 15 stars determined to be accreting (as defined in $\S 3$ ), shown in order of decreasing spectral type. 


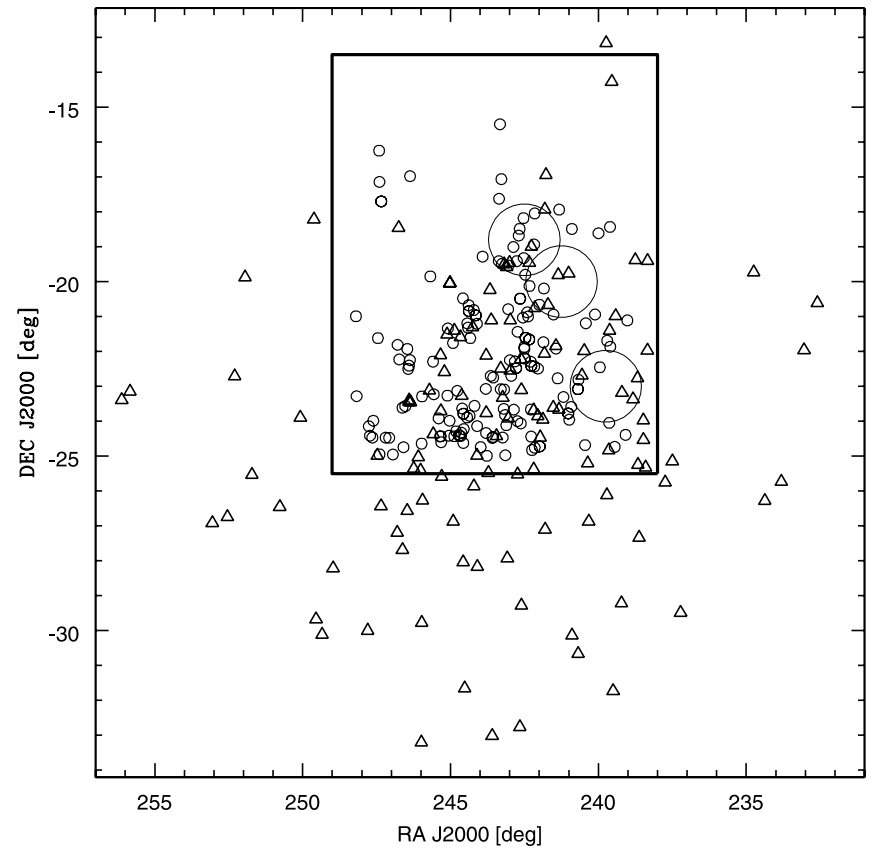

FIG. 6.-Spatial distribution of the 120 known Hipparcos members of USco (open triangle) shown with stars observed spectroscopically by us that were determined to be new members (open circles). The Quest-2 survey area is boxed in black. The approximate location and size of the $2 \mathrm{dF}$ fields observed by Preibisch et al. (2002) are shown as large circles. accreting between 1 and 5 Myr. This conclusion is consistent with a median accretion lifetime of $\sim 2-3 \mathrm{Myr}$ for higher mass stars (Haisch et al. 2001; Hillenbrand 2005).

\section{SPATIAL DISTRIBUTION OF LOW-MASS STARS}

Figure 6 shows the two-dimensional spatial distribution of the 120 high-mass members of USco identified in the Hipparcos survey (de Zeeuw et al. 1999). This sample represents the complete population of known members more massive than $\sim 1 M_{\odot}$. The density of high-mass stars is roughly constant from $237^{\circ} \lesssim \alpha \lesssim$ $249^{\circ}$ and peaks at $\delta \sim-24^{\circ}$. As can be seen, despite the large area of the Quest-2 survey, it still encompassed only the central $\sim 13^{\circ}$ in right ascension and the northern $\sim 12^{\circ}$ in declination of the association.

In general, the low-mass PMS stars presented here share a common spatial distribution with the high-mass Hipparcos members. Efforts to observe northwest of the Hipparcos stars largely yielded reddened field dwarfs rather than young association members. To correct for bias in the spatial area we observed spectroscopically, we first computed the percent of photometric candidates that we took spectra of, in $1^{\circ}$ bins, as a function of right ascension and declination. We found that we observed spectroscopically a maximum of $\sim 25 \%$ of the photometric candidates within a given degree-wide spatial bin. We thus corrected every bin to a uniform $25 \%$ of candidates observed and calculated the number of members we would have detected assuming that we had observed $25 \%$ of the photometric candidates in each bin and that the percentage of identified members relative to the number of stars observed spectroscopically at each spatial bin would remain unchanged. Figure 7 shows the resultant one-dimensional spatial distributions
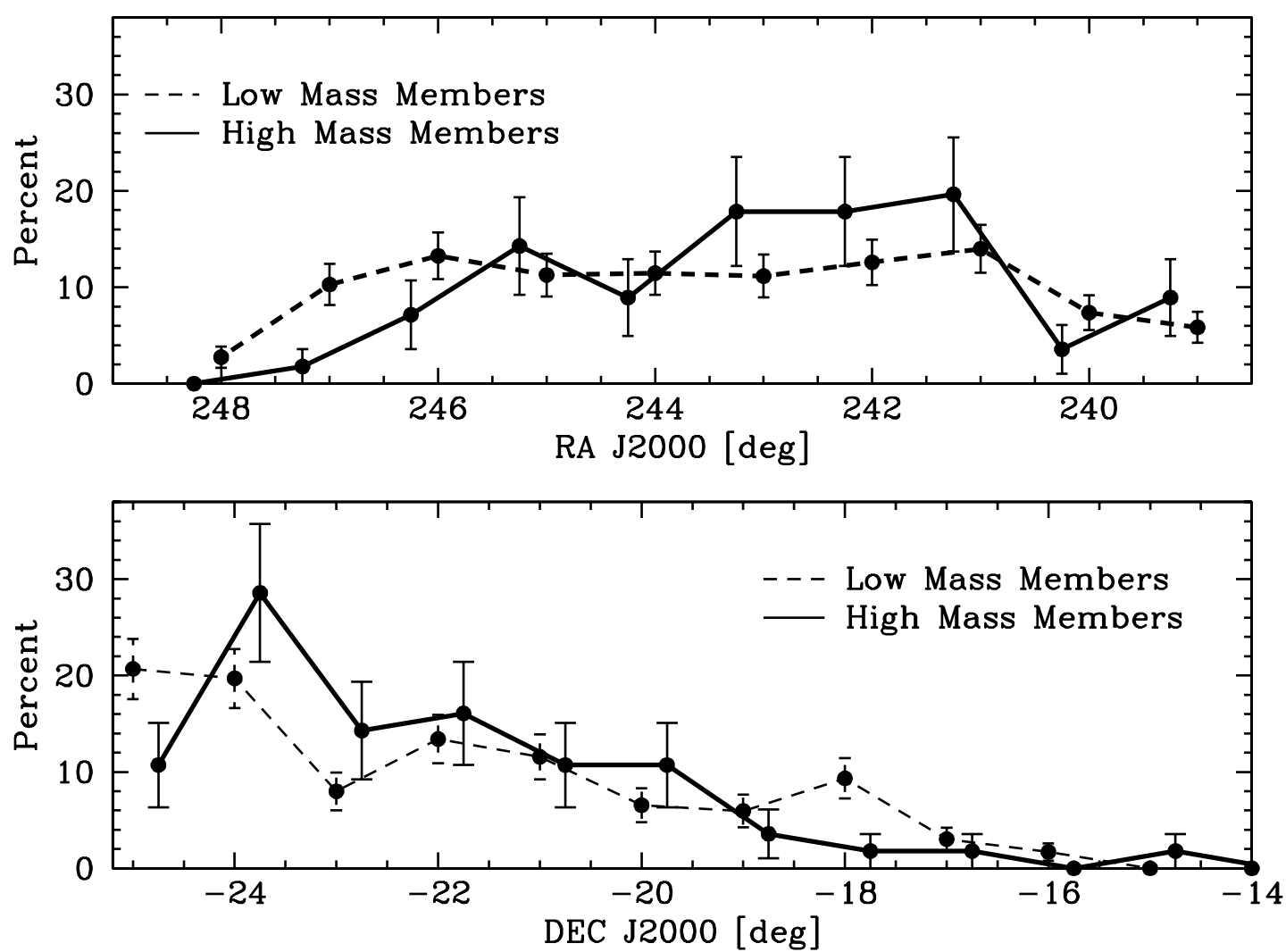

FIG. 7.-Top: Percentage of the low-mass stars $\left(M \lesssim 0.6 M_{\odot}\right)$ discovered in this work (corrected to a uniform $25 \%$ of candidates observed across all values) that lie at a given right ascension (dashed line), together with the same information for the 56 high-mass Hipparcos stars $\left(M \gtrsim 1 M_{\odot}\right.$ ) found in the Quest-2 survey area (solid line). Bottom: Same information as a function of declination. In both panels, the coordinates of the high-mass star bins have been shifted by $0.25^{\circ}$ for ease of comparison between the high- and low-mass samples. Error bars assume Poisson statistics. 
for the low-mass association members discussed here, together with those for the 56 Hipparcos stars that fall within our survey area. We conclude that the density of low-mass association members found in the Quest-2 survey is roughly uniform in right ascension and peaks at $\delta \sim-25^{\circ}$ with stellar densities falling off beyond these values. We find no evidence for spatial segregation by mass in USco within the northern portion of the association.

\section{AGE AND MASS DISTRIBUTIONS}

\subsection{H-R Diagram for New USco Members}

In this section we combine the spectral type and photometry of each new member to derive values for its luminosity and effective temperature and place it on an $\mathrm{H}-\mathrm{R}$ diagram. As described in $\S 2.1$, the final Quest-2 photometry is not on a standard magnitude system. Thus, because of the reliability and uniformity of 2MASS, we chose to use $J$-band magnitudes and $(J-H)$ colors to derive luminosities. An empirical fit to $\mathrm{BC}_{J}$ as a function of spectral type was determined from the observational data of Leggett et al. (1996, 2002) (spectral types M1-M6.5 and M6-L3, respectively). We derived intrinsic colors, extinction, and effective temperatures using the methods described in Slesnick et al. (2004).

In Figure 8 we present an H-R diagram for the 145 low-mass members of USco that we identified, shown with PMS model tracks and isochrones. The most commonly used PMS models for low-mass stars and brown dwarfs are those derived by D'Antona \& Mazzitelli (1997, hereafter DM97) and Baraffe et al. (1998), which differ primarily in their atmospheric approximations and treatment of convection. Both models suggest similar mass ranges for our data of $0.02 M_{\odot}<M<0.2 M_{\odot}$, although predicted masses for individual objects can vary by up to $0.09 M_{\odot}$ $(60 \%)$. We have found that the slope of the DM97 isochrones provides a reasonable match to the derived $\mathrm{H}-\mathrm{R}$ diagram, whereas the Baraffe et al. (1998) models predict systematically younger stars at lower masses. A similar result was also noted by Hillenbrand et al. (2008). These authors compared the slope of six different theoretical PMS isochrones as a function of binary fraction and found that, assuming an intermediate binary fraction consistent with observations (e.g., Kraus et al. 2008), the DM97 models provide the best match to the observed H-R diagram slope for stars in USco. Thus, for the remainder of this work, we use the DM97 mass tracks and isochrones to derive mass and age for stars in our sample. All derived quantities are given in Table 2.

\subsection{Age Distribution of the Low-Mass Population in USco}

Literal interpretation of the derived H-R diagram (Fig. 8) reveals a population with median age of $\sim 4.1$ Myr and a continuous spread of ages over $\gtrsim 10$ Myr. While this result may be real, the continuous nature of the observed age distribution in USco could also be produced from uncertainties in observed parameters. Unlike the H-R diagram, the observed surface gravity-sensitive spectral features do not show evidence for a large spread in age between association members. Figure 9 shows spectra of two stars with spectral type M5 identified in the survey. The top spectrum is that of the "youngest" M5 star observed spectroscopically at Palomar (SCH J16054416-21550566), 2.6 Myr old based on its location on the H-R diagram (see also Table 2); the bottom spectrum is of the "oldest" M5 star observed at Palomar $\mathrm{SCH}$ J16162599-21122315), 14.4 Myr based on its location on the H-R diagram. As discussed in SCH06 and Slesnick (2007), young stars less than a few megayears old exhibit systematically less $\mathrm{Na}$ I ( 28190) absorption than do intermediate-age stars ( 5-10 Myr) due to their lower surface gravity (see Fig. 10 of Paper I and Fig. 6

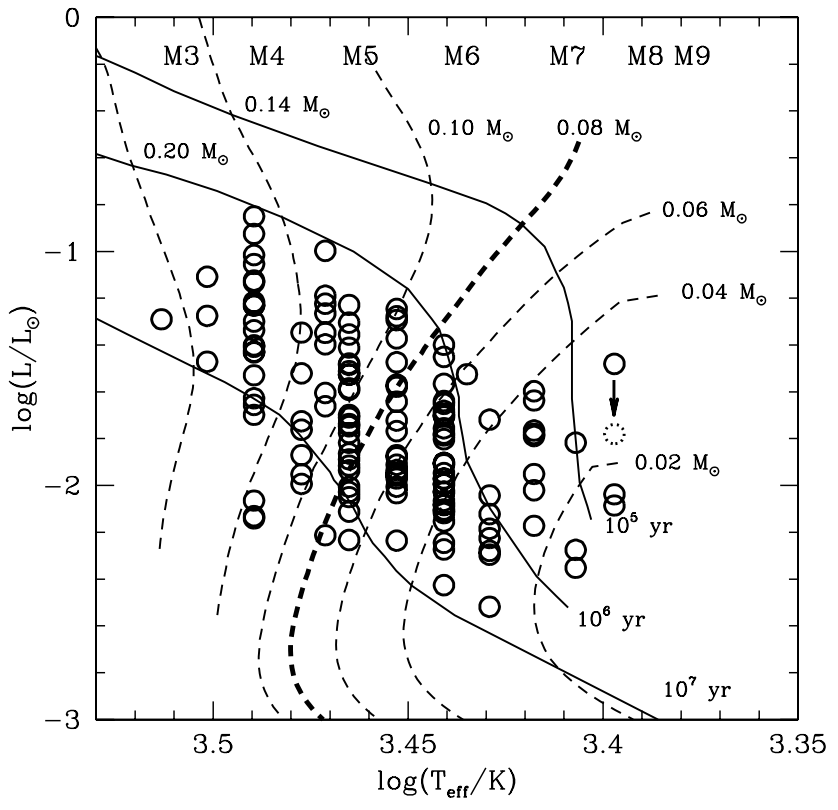

FIG. 8.- H-R diagram for new PMS objects found in the USco region, shown with model tracks and isochrones of DM97. The sample is consistent with an age of $\sim 5 \mathrm{Myr}$ and contains masses spanning the brown dwarf to stellar regimes. The arrow plus dotted symbol indicates where the star SCH J16224384-19510575 would sit in the H-R diagram as a single star if it is an unresolved, equal-mass binary (see $\S 4.2$ in Paper I).

of SCH06). Thus, if the derived ages from the H-R diagram are correct, the spectrum of the 14.4 Myr old star should have noticeably stronger $\mathrm{Na}$ I absorption. For example, an M5 star at 1-5 Myr would have a Na-8190 index 5\%-10\% larger than that observed for an M5 star at 10 Myr. The spectra presented in Figure 9 are nearly identical (and have near-identical measured Na-8190 indices of 0.89 and 0.90 ). Based on analysis of spectral features, we classified these two stars as being roughly the same age. However, based on the differences in observed luminosity, interpreting the $\mathrm{H}-\mathrm{R}$ diagram literally, one would infer an age spread of $>10 \mathrm{Myr}$ between the two stars.

\subsubsection{Comparison of the Observed Age \\ Distribution to a Coeval Population}

Due to the discrepancy between stellar ages derived from the H-R diagram compared to those inferred from spectral surface gravity signatures, we sought to determine the statistical significance of the observed spread in ages for stars on the H-R diagram. Effective temperatures and luminosities shown in Figure 8 are derived from observed $J$ magnitudes, $J-H$ colors, and spectral types. The age and mass of a star are inferred from effective temperature and luminosity using a set of theoretical isochrones and mass tracks. Thus, uncertainties in measured photometry or spectral type are propagated into uncertainties in mass and age. Variations in distance and binarity can cause additional uncertainty in luminosity and hence quantities derived from the H-R diagram.

We explore first the possibility that the USco population could be coeval and that the apparent age spread in the H-R diagram is a result of observational uncertainties combined with association depth and binarity effects. Using a similar method to that used in this work, Preibisch \& Zinnecker (1999) compute an age of 5 Myr for the intermediate-mass members of USco. We used Monte Carlo techniques to generate a coeval population of $5 \mathrm{Myr}$ old stars and brown dwarfs at a distance of $145 \mathrm{pc}$. The input spectral type distribution was selected to mirror that of our observed 
TABLE 2

Derived Quantities for New USco Members

\begin{tabular}{|c|c|c|c|c|}
\hline ID & $\log \left(T_{\text {eff }} / \mathrm{K}\right)$ & $\log \left(L / L_{\odot}\right)$ & $\log (\text { age } / y r)^{a}$ & $M / M_{\odot}^{\mathrm{a}}$ \\
\hline SCH J15560497-21064632 ..... & 3.41 & -2.17 & 5.46 & 0.03 \\
\hline SCH J15561978-24232936.... & 3.47 & -1.87 & 7.10 & 0.12 \\
\hline SCH J15574757-24441236..... & 3.49 & -1.69 & 7.09 & 0.16 \\
\hline SCH J15582337-21515908_... & 3.47 & -1.39 & 6.54 & 0.13 \\
\hline SCH J15582566-18260865 ..... & 3.44 & -1.65 & 6.41 & 0.06 \\
\hline SCH J15583162-24025411 .... & 3.47 & -1.76 & 7.00 & 0.12 \\
\hline SCH J15584812-21413426..... & 3.45 & -1.95 & 6.75 & 0.06 \\
\hline SCH J15594802-22271650_.... & 3.40 & -2.27 & 5.35 & 0.02 \\
\hline SCH J15595868-18365205 .... & 3.45 & -1.88 & 6.71 & 0.06 \\
\hline SCH J16002669-20563190_... & 3.47 & -1.95 & 7.17 & 0.11 \\
\hline SCH J16014156-21113855 ..... & 3.49 & -1.52 & 6.88 & 0.16 \\
\hline SCH J16014768-24410152_... & 3.46 & -2.11 & 7.06 & 0.07 \\
\hline SCH J16024143-22484204_.... & 3.45 & -1.76 & 6.64 & 0.07 \\
\hline SCH J16024576-23045102 .... & 3.46 & -1.52 & 6.61 & 0.11 \\
\hline SCH J16033470-18293060_.... & 3.46 & -1.48 & 6.57 & 0.11 \\
\hline SCH J16034029-23352386..... & 3.49 & -1.40 & 6.70 & 0.17 \\
\hline SCH J16035651-23572517 .... & 3.47 & -1.99 & 7.21 & 0.11 \\
\hline SCH J16040453-23463795 .... & 3.49 & -1.13 & 6.35 & 0.16 \\
\hline SCH J16044303-23182620.... & 3.42 & -2.12 & 5.96 & 0.03 \\
\hline SCH J16051829-17562092 _.... & 3.49 & -1.12 & 6.34 & 0.16 \\
\hline SCH J16053077-22462016.... & 3.44 & -2.10 & 6.75 & 0.04 \\
\hline SCH J16054416-21550566..... & 3.46 & -1.30 & 6.42 & 0.12 \\
\hline SCH J16060391-20564497..... & 3.41 & -2.02 & 5.44 & 0.03 \\
\hline SCH J16072239-20115852 ..... & 3.45 & -1.57 & 6.54 & 0.08 \\
\hline SCH J16072640-21441727.... & 3.44 & -2.42 & 6.94 & 0.03 \\
\hline SCH J16075565-24432714..... & 3.45 & -2.00 & 6.78 & 0.06 \\
\hline SCH J16075850-20394890..... & 3.44 & -1.99 & 6.68 & 0.04 \\
\hline SCH J16081081-22294303 .... & 3.46 & -1.58 & 6.66 & 0.11 \\
\hline SCH J16083646-24453053 ..... & 3.50 & -1.10 & 6.41 & 0.18 \\
\hline SCH J16083658-18024994_.... & 3.42 & -1.71 & 5.64 & 0.05 \\
\hline SCH J16084058-22255726.... & 3.49 & -2.14 & 7.60 & 0.13 \\
\hline SCH J16084170-18561077 .... & 3.44 & -1.45 & 6.09 & 0.07 \\
\hline SCH J16085870-24493641 .... & 3.49 & -1.40 & 6.71 & 0.17 \\
\hline SCH J16090451-22245259..... & 3.41 & -1.78 & 5.39 & 0.04 \\
\hline SCH J16090511-24262843 .... & 3.49 & -1.21 & 6.45 & 0.16 \\
\hline SCH J16090771-23395430.... & 3.46 & -1.35 & 6.46 & 0.12 \\
\hline SCH J16090883-22174699..... & 3.46 & -1.71 & 6.76 & 0.10 \\
\hline SCH J16091837-20073523 .... & 3.40 & -1.81 & 5.01 & 0.02 \\
\hline SCH J16092137-21393452.... & 3.45 & -1.37 & 6.39 & 0.10 \\
\hline SCH J16093018-20595409..... & 3.44 & -2.15 & 6.77 & 0.04 \\
\hline SCH J16093707-20525337..... & 3.47 & -1.66 & 6.81 & 0.12 \\
\hline SCH J16095217-21362826..... & 3.41 & -1.63 & 5.36 & 0.04 \\
\hline SCH J16095307-19481704..... & 3.44 & -1.68 & 6.43 & 0.06 \\
\hline SCH J16095695-22120300_.... & 3.45 & -2.03 & 6.80 & 0.06 \\
\hline SCH J16095991-21554293 ..... & 3.42 & -2.27 & 6.16 & 0.03 \\
\hline SCH J16100129-21522466.... & 3.45 & -1.64 & 6.57 & 0.08 \\
\hline SCH J16100541-19193636.... & 3.44 & -2.10 & 6.74 & 0.04 \\
\hline SCH J16100751-18105666..... & 3.44 & -1.69 & 6.44 & 0.06 \\
\hline SCH J16101190-21015540.... & 3.45 & -1.91 & 6.73 & 0.06 \\
\hline SCH J16102990-24035024..... & 3.47 & -1.72 & 6.96 & 0.13 \\
\hline SCH J16103525-20291714_.... & 3.46 & -1.59 & 6.66 & 0.11 \\
\hline SCH J16103876-18292353 ..... & 3.44 & -1.99 & 6.68 & 0.04 \\
\hline SCH J16104635-18405996..... & 3.47 & -1.34 & 6.53 & 0.14 \\
\hline SCH J16105500-21261422 .... & 3.44 & -1.63 & 6.40 & 0.06 \\
\hline SCH J16105727-23595416.... & 3.49 & -1.62 & 7.01 & 0.16 \\
\hline SCH J16110144-19244914_.... & 3.46 & -1.86 & 6.88 & 0.09 \\
\hline SCH J16110739-22285027 ..... & 3.43 & -1.52 & 5.75 & 0.06 \\
\hline SCH J16111711-22171749 ..... & 3.40 & -2.35 & 5.38 & 0.02 \\
\hline SCH J16112629-23400611 .... & 3.45 & -1.93 & 6.74 & 0.06 \\
\hline SCH J16112959-19002921 _.... & 3.44 & -1.90 & 6.61 & 0.05 \\
\hline SCH J16114735-22420649 ..... & 3.46 & -1.88 & 6.90 & 0.09 \\
\hline SCH J16115737-22150691 .... & 3.46 & -2.02 & 7.00 & 0.08 \\
\hline SCH J16121044-19322708..... & 3.46 & -1.41 & 6.51 & 0.12 \\
\hline SCH J16121188-20472698..... & 3.42 & -2.04 & 5.87 & 0.03 \\
\hline SCH J16122764-24064850..... & 3.41 & -1.76 & 5.39 & 0.04 \\
\hline
\end{tabular}

TABLE 2-Continued

\begin{tabular}{|c|c|c|c|c|}
\hline ID & $\log \left(T_{\mathrm{eff}} / \mathrm{K}\right)$ & $\log \left(L / L_{\odot}\right)$ & $\log (\text { age } / y r)^{a}$ & $M / M_{\odot}^{\mathrm{a}}$ \\
\hline SCH J16123459-24583447.... & 3.47 & -1.34 & 6.49 & 0.13 \\
\hline SCH J16123758-23492340..... & 3.44 & -2.11 & 6.75 & 0.04 \\
\hline SCH J16124506-23053043 ..... & 3.45 & -1.95 & 6.75 & 0.06 \\
\hline SCH J16124692-23384086.... & 3.44 & -2.02 & 6.70 & 0.04 \\
\hline SCH J16125723-24280145 ..... & 3.49 & -1.65 & 7.04 & 0.16 \\
\hline SCH J16130306-19293234 ..... & 3.45 & -1.87 & 6.70 & 0.06 \\
\hline SCH J16130764-17035233 ..... & 3.45 & -1.97 & 6.76 & 0.06 \\
\hline SCH J16131212-23050329..... & 3.42 & -2.22 & 6.09 & 0.03 \\
\hline SCH J16131857-15293460..... & 3.47 & -1.18 & 6.34 & 0.13 \\
\hline SCH J16132576-17373542 ..... & 3.49 & -1.43 & 6.74 & 0.17 \\
\hline SCH J16132809-19245288 ..... & 3.44 & -1.70 & 6.45 & 0.06 \\
\hline SCH J16141351-22445788 ..... & 3.49 & -1.42 & 6.74 & 0.17 \\
\hline SCH J16141484-24270844 ..... & 3.41 & -1.59 & 5.35 & 0.04 \\
\hline SCH J16141974-24284053 ..... & 3.44 & -2.06 & 6.72 & 0.04 \\
\hline SCH J16143286-22421358..... & 3.42 & -2.29 & 6.18 & 0.03 \\
\hline SCH J16150524-24593542 ..... & 3.46 & -1.52 & 6.61 & 0.11 \\
\hline SCH J16151115-24201556 ..... & 3.44 & -2.24 & 6.82 & 0.04 \\
\hline SCH J16151360-23042637..... & 3.42 & -2.51 & 6.89 & 0.03 \\
\hline SCH J16153915-19170073 ..... & 3.47 & -0.99 & 6.08 & 0.13 \\
\hline SCH J16155508-24443677 ..... & 3.44 & -1.90 & 6.61 & 0.05 \\
\hline SCH J16162396-24083016..... & 3.46 & -1.77 & 6.81 & 0.09 \\
\hline SCH J16162599-21122315 ..... & 3.46 & -2.23 & 7.16 & 0.07 \\
\hline SCH J16163504-20575551 ..... & 3.45 & -1.47 & 6.48 & 0.09 \\
\hline SCH J16164538-23334143 ..... & 3.46 & -2.05 & 7.01 & 0.08 \\
\hline SCH J16165160-20485398..... & 3.49 & -1.29 & 6.56 & 0.16 \\
\hline SCH J16171901-21371312.... & 3.45 & -1.94 & 6.75 & 0.06 \\
\hline SCH J16172504-23503799..... & 3.46 & -1.93 & 6.93 & 0.08 \\
\hline SCH J16173105-20504715 ..... & 3.41 & -1.78 & 5.39 & 0.04 \\
\hline SCH J16173238-20403653 ..... & 3.44 & -2.27 & 6.84 & 0.04 \\
\hline SCH J16173788-21191618 ..... & 3.49 & -1.33 & 6.61 & 0.16 \\
\hline SCH J16174368-21115536 ..... & 3.49 & -2.13 & 7.59 & 0.13 \\
\hline SCH J16174540-23533618 ..... & 3.44 & -2.08 & 6.73 & 0.04 \\
\hline SCH J16181201-24133263 ..... & 3.46 & -1.47 & 6.57 & 0.11 \\
\hline SCH J16181567-23470847..... & 3.45 & -1.24 & 6.26 & 0.10 \\
\hline SCH J16181601-24372688..... & 3.49 & -1.05 & 6.26 & 0.16 \\
\hline SCH J16181906-20284815 ..... & 3.47 & -1.22 & 6.37 & 0.13 \\
\hline SCH J16182501-23381068..... & 3.46 & -1.81 & 6.85 & 0.09 \\
\hline SCH J16183144-24195229..... & 3.42 & -2.18 & 6.04 & 0.03 \\
\hline SCH J16183620-24253332 ..... & 3.49 & -1.23 & 6.47 & 0.16 \\
\hline SCH J16185038-24243205 ..... & 3.46 & -1.91 & 6.92 & 0.08 \\
\hline SCH J16190473-23075283 ..... & 3.45 & -1.72 & 6.62 & 0.07 \\
\hline SCH J16191521-24172429..... & 3.49 & -1.22 & 6.47 & 0.16 \\
\hline SCH J16192994-24255414..... & 3.49 & -0.85 & 6.05 & 0.15 \\
\hline SCH J16193976-21453527..... & 3.44 & -1.80 & 6.53 & 0.05 \\
\hline SCH J16200756-23591522 ..... & 3.44 & -1.75 & 6.49 & 0.05 \\
\hline SCH J16201318-24250155 ..... & 3.49 & -2.06 & 7.52 & 0.14 \\
\hline SCH J16202127-21202923 ..... & 3.44 & -1.90 & 6.61 & 0.05 \\
\hline SCH J16202523-23160347..... & 3.45 & -2.23 & 6.94 & 0.05 \\
\hline SCH J16211564-24361173 ..... & 3.50 & -1.47 & 6.93 & 0.19 \\
\hline SCH J16211922-24255250 .... & 3.49 & -1.01 & 6.22 & 0.16 \\
\hline SCH J16212490-24261446..... & 3.50 & -1.27 & 6.64 & 0.19 \\
\hline SCH J16213591-23550341 ..... & 3.44 & -2.02 & 6.70 & 0.04 \\
\hline SCH J16221577-23134936.... & 3.44 & -2.08 & 6.73 & 0.04 \\
\hline SCH J16222156-22173094_.... & 3.46 & -2.00 & 6.98 & 0.08 \\
\hline SCH J16224384-19510575 ..... & 3.39 & -1.48 & $\cdots$ & 0.04 \\
\hline SCH J16235158-23172740.... & 3.39 & -2.03 & $\ldots$ & 0.02 \\
\hline SCH J16235474-24383211 ..... & 3.44 & -1.70 & 6.45 & 0.06 \\
\hline SCH J16252862-16585055 ..... & 3.39 & -2.08 & $\ldots$ & 0.02 \\
\hline SCH J16252968-22145448 ..... & 3.46 & -1.73 & 6.78 & 0.10 \\
\hline SCH J16253671-22242887..... & 3.41 & -1.95 & 5.42 & 0.03 \\
\hline SCH J16254319-22300300..... & 3.46 & -1.74 & 6.79 & 0.10 \\
\hline SCH J16255064-21554577 ..... & 3.47 & -2.21 & 7.28 & 0.08 \\
\hline SCH J16260630-23340375 ..... & 3.45 & -1.56 & 6.53 & 0.08 \\
\hline SCH J16263026-23365552 ..... & 3.44 & -1.77 & 6.51 & 0.05 \\
\hline SCH J16265619-22135224.... & 3.44 & -1.94 & 6.64 & 0.05 \\
\hline SCH J16270940-21484591 ..... & 3.47 & -1.52 & 6.73 & 0.14 \\
\hline
\end{tabular}


TABLE 2-Continued

\begin{tabular}{|c|c|c|c|c|}
\hline ID & $\log \left(T_{\text {eff }} / \mathrm{K}\right)$ & $\log \left(L / L_{\odot}\right)$ & $\log (\mathrm{age} / \mathrm{yr})^{\mathrm{a}}$ & $M / M_{\odot}^{\mathrm{a}}$ \\
\hline SCH J16274801-24571371.... & 3.46 & -1.69 & 6.75 & 0.10 \\
\hline SCH J16281810-24283619..... & 3.44 & -1.39 & 5.95 & 0.08 \\
\hline SCH J16284706-24281413..... & 3.44 & -1.78 & 6.51 & 0.05 \\
\hline SCH J16292211-17420937..... & 3.47 & -1.60 & 6.75 & 0.12 \\
\hline SCH J16293625-24565325..... & 3.44 & -1.97 & 6.66 & 0.05 \\
\hline SCH J16293664-17084094..... & 3.47 & -1.26 & 6.41 & 0.13 \\
\hline SCH J16293934-16145647..... & 3.51 & -1.29 & 6.79 & 0.22 \\
\hline SCH J16294877-21370914..... & 3.46 & -1.51 & 6.60 & 0.11 \\
\hline SCH J16302675-23590905..... & 3.44 & -1.56 & 6.34 & 0.07 \\
\hline SCH J16303392-24280657..... & 3.49 & -0.92 & 6.13 & 0.15 \\
\hline SCH J16305349-24245439..... & 3.45 & -1.27 & 6.31 & 0.10 \\
\hline SCH J16310241-24084335..... & 3.46 & -1.22 & 6.35 & 0.12 \\
\hline SCH J16324224-23165644..... & 3.45 & -1.29 & 6.33 & 0.10 \\
\hline SCH J16324726-20593771..... & 3.44 & -1.97 & 6.66 & 0.04 \\
\hline
\end{tabular}

${ }^{a}$ Masses and ages derived from the model tracks and isochrones of DM97.

distribution of stars, with 1000 stars simulated for every star observed. For each star in the simulated population, $J$ - and $H$-band magnitudes were varied by adding random offsets drawn from a Gaussian distribution with a $1 \sigma$ deviation of $0.025 \mathrm{mag}$, corresponding to the average uncertainty in the 2MASS photometry for observed stars. To mimic the magnitude-limited data sample, we did not allow any star to be simulated below the photometric survey limits $(J=16$ and $H=15.5)$. Similarly, a random offset was added to the assumed spectral type, selected from a Gaussian distribution with $1 \sigma$ errors of 0.5 spectral subtypes corresponding to the qualitative error of the optical spectral type determinations. Simulated spectral types were rounded to the nearest 0.25 subclasses to reflect the discreet nature of spectral type classification for new members discovered in our work. Using the new spectral type, for each simulated star, we rederived the expected effective temperature, bolometric correction, and intrinsic $J-H$ color using the methods described in Slesnick et al. (2004).

The maximum distance spread (derived from secular parallax measurements) among members of the association with Hipparcos measurements is $50 \mathrm{pc}$ (Preibisch et al. 2002; de Bruijne 1999). In the simulation, we assumed a uniform spatial distribution over a box of this depth centered at $145 \mathrm{pc}$. A $33 \%$ binary fraction for stars across all simulated spectral types (M3-M8) was assumed, consistent with observational results of the binary frequency for low-mass members of USco (Kraus et al. 2005, 2008). All observed low-mass binaries in USco have near equal masses ( $m_{\text {secondary }} / m_{\text {primary }} \gtrsim 0.6$; Kraus et al. 2005 ); thus, the assumption was made that all binaries were composed of two equalmass stars. This assumption is somewhat liberal in that it will produce the largest possible dispersion in luminosity.

We compare quantitatively results of this simulation to the observed data in Figure 10. The red shaded histogram shows the age distribution derived from the Monte Carlo simulation, overplotted with a histogram of ages for the data (black hatched histogram). Both histograms have been normalized to unity at the peak for comparison, and all stars with $\log T_{\text {eff }}<3.4$ (beyond which interpolation of the isochrones becomes unreliable) have been excluded from the data and the model results. The widths of the distributions are remarkably similar, given the simplistic nature of the Monte Carlo simulation. For the simulated association, we find a mean apparent age of $\log ($ age $)=6.51 \pm 0.40$, whereas for the data (not including the three M8 stars) we find a mean age of $\log (\mathrm{age})=6.53 \pm 0.47$. The data and the model differ significantly at the distribution tails, which is most likely caused either
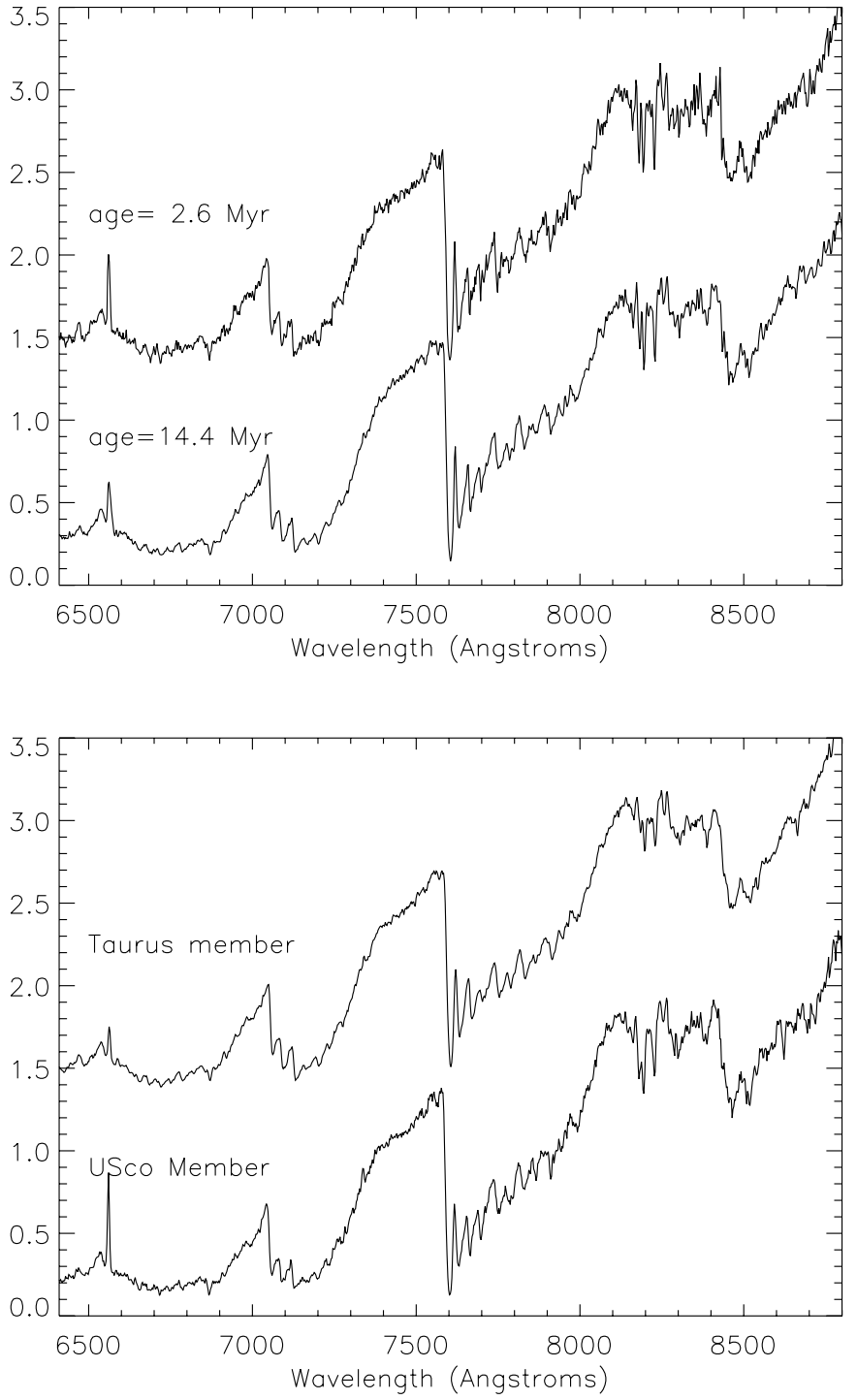

FIG. 9.-Top: Spectra of two stars with spectral type M5 found in the USco survey. The top spectrum is of the youngest (based on analysis of the H-R diagram) M5 star observed spectroscopically at Palomar, $\sim 2.6$ Myr old; the bottom spectrum is of the oldest (based on analysis of the H-R diagram) M5 star observed at Palomar, $\sim 14.4$ Myr. However, these stars have near-identical spectra and, based on analysis of the strength of the surface gravity-sensitive Na I line (28190), appear to be the same age. Bottom: Spectra of known members of Taurus and USco with spectral type M5, for comparison. The two stars have measured Na-8190 indices that are different by $\sim 9 \%$ and can be easily distinguished from each other visually.

by an incorrect assumption of one of the model parameters or by an inherent problem with the theoretical isochrones at very low masses (Hillenbrand \& White 2004). To quantitatively assess how well the model reproduces the bulk of the data, excluding the distribution tails, we applied a $\chi^{2}$ test of the central peaks of the distributions from $\log$ (age) $=6.0$ to 7.4. This test yields a $\sim 53 \%$ probability that the two distributions could have been drawn from the same population. Thus, given the uncertainties, the data are consistent with most stars in USco forming via a single burst $~ 5 \mathrm{Myr}$ ago.

This result is somewhat surprising given the large extent of the association ( $\sim 35 \mathrm{pc}$ across). Assuming a sound speed of $c_{s}=0.2 \mathrm{~km} \mathrm{~s}^{-1}$ consistent with a $T=10 \mathrm{~K}$ molecular cloud, and assuming that the stars formed close to where they are observed today, the sound crossing time for the parental molecular cloud is $\sim 85 \mathrm{Myr}$. Thus, one end of the cloud could not 


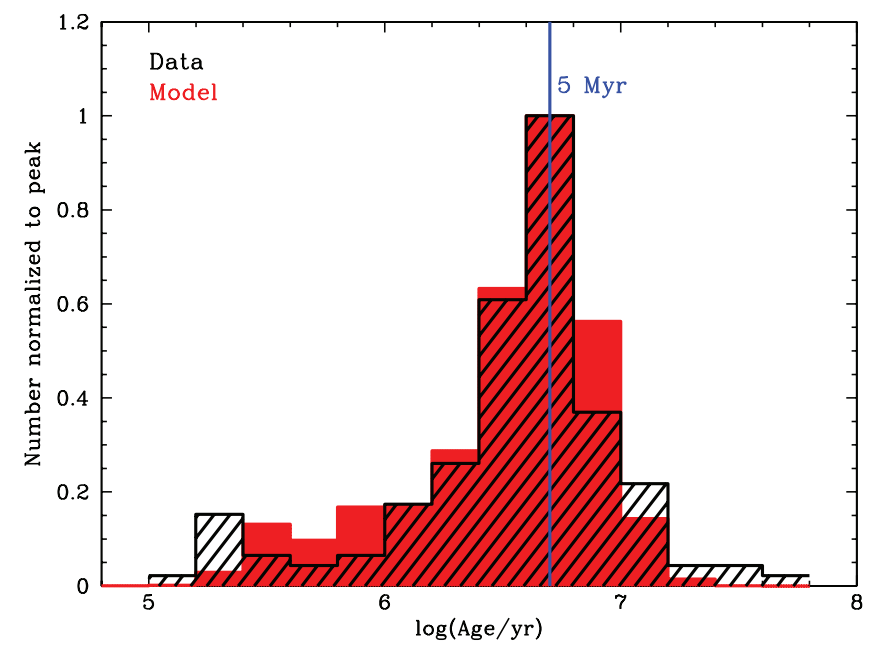

FIG. 10.- Red shaded histogram: Age distribution derived from the Monte Carlo simulation, overplotted with a histogram of ages for the data (black hatched histogram). Both histograms have been normalized to unity at the peak for comparison, and stars having $\log T_{\text {eff }}<3.4$ (beyond which interpolation of the isochrones becomes unreliable) have been excluded from the data and the model results. The widths of the distributions are remarkably similar, given the simplistic nature of the Monte Carlo simulation.

have "communicated" to the other in time to create a simultaneous burst of star formation. This result does not, however, rule out the possibility that star formation happened simultaneously throughout the extent of the cloud because every part independently reached the threshold for star formation at the same time. Another scenario is that the stars formed much closer together and have since spread to their current positions. However, this possibility can be ruled out from simple arguments. The velocity dispersion of the Hipparcos members is $\sim 1.3 \mathrm{~km} \mathrm{~s}^{-1}$ (de Bruijne 1999). In $5 \mathrm{Myr}$, the furthest an association member could travel at a speed of $1.3 \mathrm{~km} \mathrm{~s}^{-1}$ is $\sim 6.5 \mathrm{pc}$, less than half the radius of the current association.

A similar discrepancy between a small observed age spread and the large spatial extent of the association has also been noted in the literature for USco's intermediate- and high-mass members (Preibisch \& Zinnecker 1999). To explain the disparity, Preibisch \& Zinnecker (1999) (and later Preibisch \& Zinnecker 2007) proposed a scenario in which star formation in USco was triggered by an external event in the form of a supernova explosion in the neighboring Upper Centaurus-Lupus association ( $\sim 70$ pc away and $\sim 17$ Myr old). They argue based on the structure and kinematics of large $\mathrm{H}_{\mathrm{I}}$ loops surrounding Sco Cen that such an event is evidenced to have occurred $\sim 12 \mathrm{Myr}$ ago. If true, the explosion would have driven a shock wave that would have reached USco about $\sim 5$ Myr ago, consistent with the inferred age of USco's stellar population. Our results may imply a similar small age spread with an association age $\sim 5 \mathrm{Myr}$ and large spatial extent (see $\S 4$ ) for USco's lowest mass stars and substellar members. While our results do not prove the Preibisch \& Zinnecker (1999) hypothesis true, they do give support to the plausibility of the hypothesis and extend its validity to even the lowest mass association members.

\subsubsection{Comparison of the Observed Age Distribution to a Uniform Distribution}

From the simulation discussed in $\S 5.2 .1$, we have determined that the observed age distribution in USco is consistent with all stars forming in a single burst 5 Myr ago. We explore also the maximum age spread that can be inferred from our data assum-

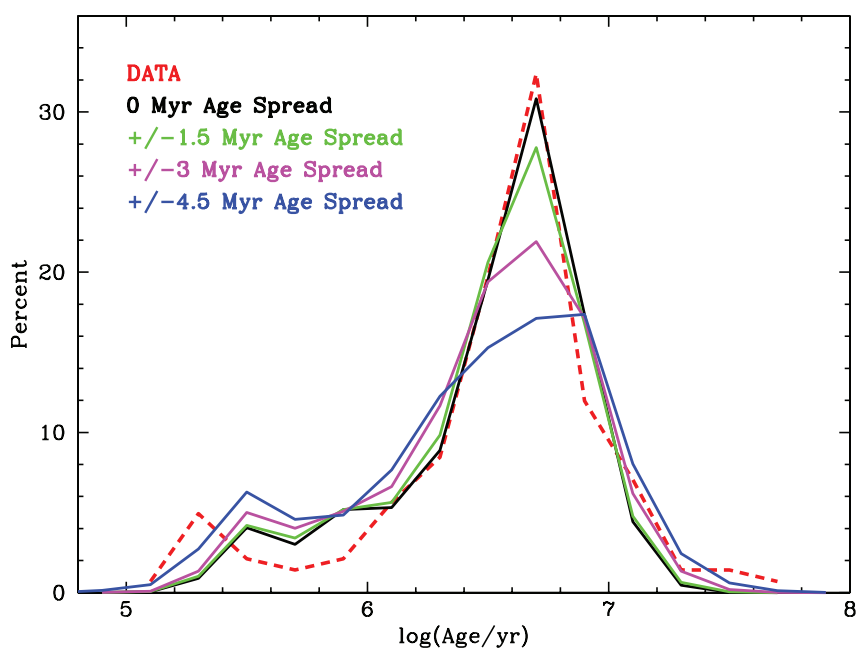

FIG. 11.-Red dashed line: Age distribution derived from spectral data, plotted as percentage of the total stars that are at a certain age. Colored solid lines: Same information for results from the coeval model (black), together with results from model age distributions of $5 \pm 1.5 \mathrm{Myr}$ (green), $5 \pm 3 \mathrm{Myr}$ (magenta), and $5 \pm 4.5 \mathrm{Myr}$ (blue). As expected, the peak of the simulated distribution decreases and more power is seen in the wings as a larger age spread is injected into the population.

ing that the star formation rate has been constant in time. We repeated the original Monte Carlo simulation, allowing age to vary in addition to spectral type, photometric error, distance, and binarity. We began with a 5 Myr population, and for each star we added a random offset in age drawn from a uniform population between $\pm 0.5 \mathrm{Myr}, \pm 1 \mathrm{Myr}, \pm 1.5 \mathrm{Myr}, \pm 2 \mathrm{Myr}, \pm 2.5 \mathrm{Myr}$ $\pm 3 \mathrm{Myr}, \pm 3.5 \mathrm{Myr}, \pm 4 \mathrm{Myr}, \pm 4.5 \mathrm{Myr}$, or $\pm 5 \mathrm{Myr}$. This age offset had the effect of changing the starting $J$ and $H$ magnitudes. All other parameters were computed as described in $\S 5.2 .1$.

Figure 11 compares the observed age distribution derived from the H-R diagram with the results from the Monte Carlo simulation. As expected, the peak of the simulated distribution decreases and more power is seen in the wings as a larger age spread is injected into the population. We have run a $\chi^{2}$ test between central peaks of the data and the simulated model distributions between $\log ($ age $)=6$ and 7.4. We find that the computed $\chi^{2}$ probability remains high, between $50 \%$ and $60 \%$, for simulations with an age spread of $\leq \pm 2 \mathrm{Myr}$, and then falls off rapidly with probabilities of $<5 \%$ beyond \pm 3 Myr. Thus, we conclude that the observed low-mass population of USco formed in $<6 \mathrm{Myr}$, and most likely $<4$ Myr; this finding is much less than the $>10$ Myr age spread implied by literal interpretation of the H-R diagram.

\subsection{The Low-Mass IMF}

In this section we use model tracks to derive the first spectroscopic mass function for stars and brown dwarfs in USco less massive than $\sim 0.1 M_{\odot}$. The study by Preibisch et al. (2002) derived an IMF for the stellar population in the association by combining the 166 spectroscopically confirmed low-mass members $(M \sim 0.8-$ $0.1 M_{\odot}$ ) discovered via their $2 \mathrm{dF}$ survey with the 120 known Hipparcos members (constituting a complete sample of members more massive than $\sim 1 M_{\odot}$ ) and 84 X-ray-selected members $\left(M \sim 0.8-2 M_{\odot}\right.$; Preibisch \& Zinnecker 1999). The resultant mass function yielded a three-segment power-law function that was consistent with recent field star and other cluster IMF determinations above $\sim 2 M_{\odot}$, but with a $2 \sigma$ excess of stars above numbers seen in the field at lower masses.

The only previous survey that has attempted to derive an IMF for USco's substellar members is the photometric study by Lodieu 


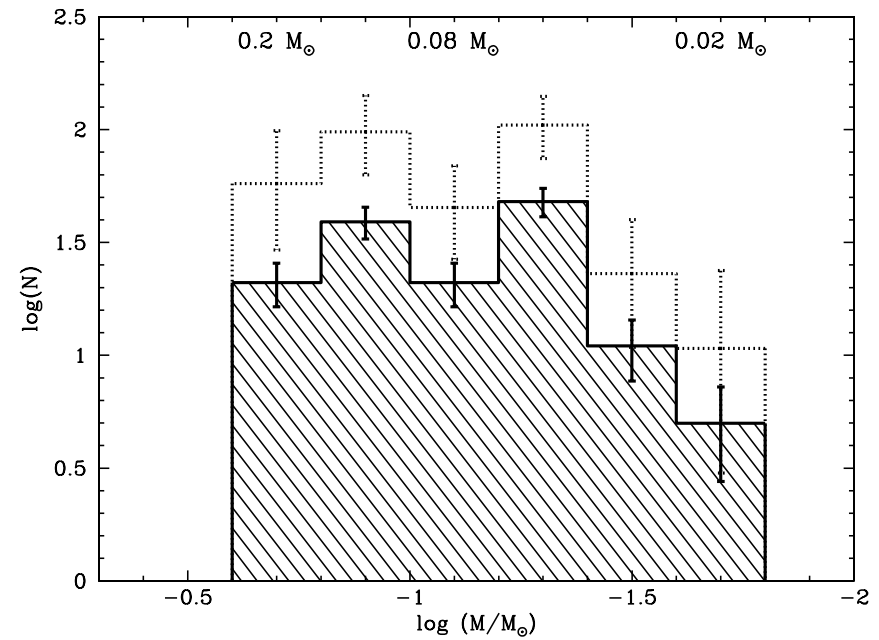

FIG. 12.-Mass function for all spectroscopically confirmed members presented in this work. The thick-lined hatched histogram indicates all stars in our spectroscopic sample; the dotted open histogram represents the same sample corrected for incomplete selection bins $(\S 5.3)$. Error bars assume Poisson statistics.

et al. (2007). These authors observed $\sim 6.5 \mathrm{deg}^{2}$ of USco at ZYJHK bands as part of the UKIRT Infrared Deep Sky Survey (UKIDSS) Galactic Cluster Survey. From these data, the authors used the $J$-band luminosity function to derive a photometric IMF in USco from 0.3 to $0.007 M_{\odot}$. They derived a mass function that was slightly flatter $\left(d N / d M \propto M^{-0.6 \pm 0.1}\right)$ than the IMF derived by Preibisch et al. (2002) for similar mass ranges $\left(d N / d M \propto M^{-0.9 \pm 0.2}\right.$ for $0.1 \leq$ $\left.M / M_{\odot}<0.6\right)$. However, as noted in Slesnick et al. (2004), photometrically derived IMFs suffer from degeneracies between mass, age, distance, extinction, and photometric excess present in the CMDs from which they are derived, and they cannot directly (only statistically) correct for field star contamination.

As was discussed in $\S 5.2$, deriving quantities from spectroscopic data placed onto an H-R diagram has its own set of uncertainties. We have already shown that known uncertainties in observable magnitudes and spectral types can produce an apparent age spread of $>10 \mathrm{Myr}$ and therefore must also determine if uncertainties in the data can produce a false spread in the observed mass distribution. To address this issue, we derived a theoretical IMF for a coeval population of stars at $5 \mathrm{Myr}$. We assumed a spectral type distribution consistent with that for our observed population and assigned each star the mass that it would have at that spectral type if it were $5 \mathrm{Myr}$ old. Examination of the difference between the two mass distributions using a K-S test reveals that the mass distributions derived from the data and the theoretical 5 Myr old population are statistically consistent with each other ( probability $=12 \%$ ). Because the mass tracks are close to vertical for low-mass stars (see Fig. 8), the dominant source of uncertainty in derived masses will come from uncertainties in temperature, arising from uncertainties in spectral type ( \pm 0.5 subtypes). Presuming that we are equally likely to misclassify a star 0.5 subtypes too early as we are to misclassify it 0.5 subtypes too late, the mass distribution will not change significantly. Thus, we conclude that the mass distribution derived from the H-R diagram is robust to observable uncertainties. We note, however, that the mass distribution is strongly affected by systematics in the theoretical models, and results will vary dependent on which sets of mass tracks are used.

In Figure 12 we show the IMF derived using the DM97 models for our spectroscopic survey of low-mass stars and brown dwarfs, covering the mass range $M \sim 0.2-0.02 M_{\odot}$. Because we have observed spectroscopically only $\sim 15 \%$ of the photometric

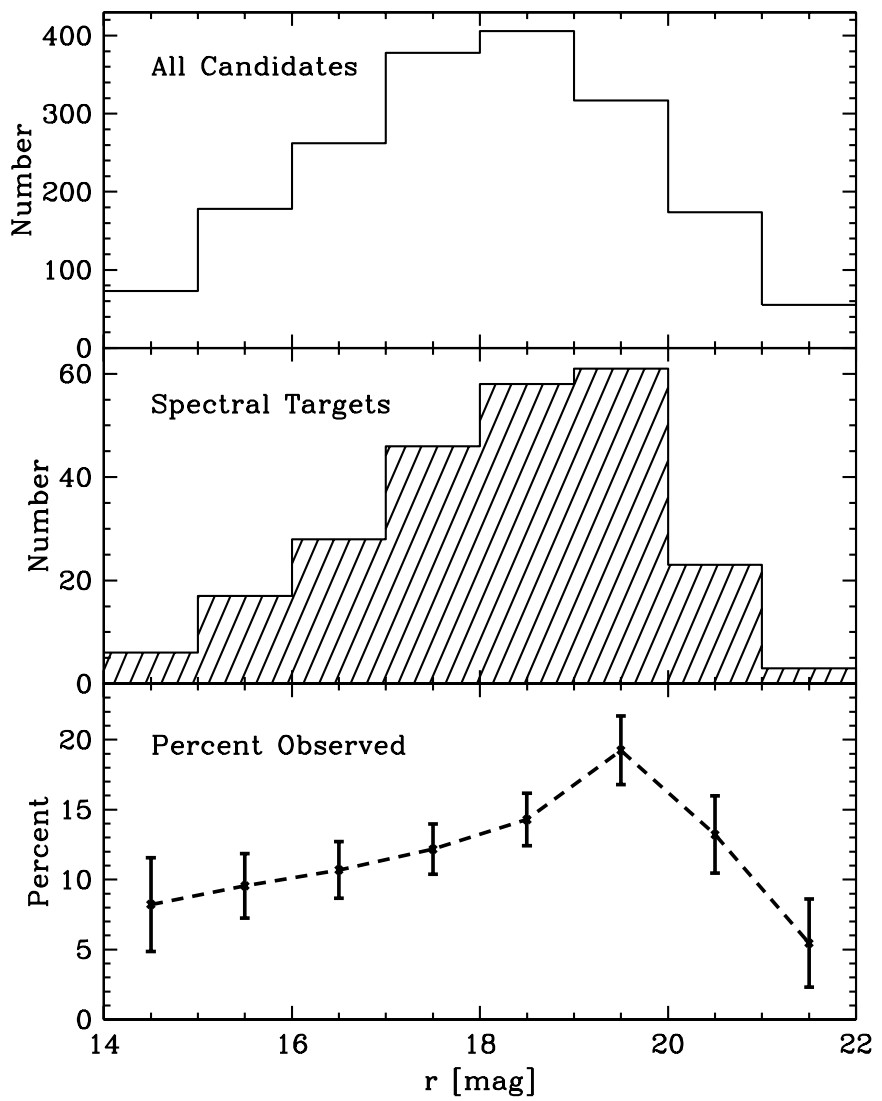

FIG. 13.- Histogram of optical CMD completeness as a function of $r$ magnitude. Bin centers correspond to $r$ magnitudes at the $1 \%$ contour line in Fig. 1. Top: Data for all photometric PMS candidates selected as in $\S 2.1$. Middle: Data for all targets observed spectroscopically. Bottom: Percent completeness with $\sqrt{N}$ error bars. Completeness peaks at $20 \%$ near $r=19.5$ mag.

PMS star candidates, before constructing a mass function, it is necessary to determine that the spectroscopic sample presented here is representative of the USco low-mass population as a whole. We use a method similar to that employed by Slesnick et al. (2004) and determine the relative completeness of our sample by computing the number of stars observed spectroscopically compared to the number of photometric candidates present in uniformly spaced bins perpendicular to the line of selection at the $1 \%$ data contour (see Fig. 1). As can be seen in Figure 13, the number of observed stars falls off considerably for stars with magnitudes $r \gtrsim 19.5$, which could be observed only under the best seeing conditions. The spectroscopic survey completeness peaks at $\sim 20 \%$. We correct the IMF to uniform completeness at this level by determining the mass distribution of stars within each magnitude bin. We then add stars to the relevant mass bins based on the fractional completeness of the magnitude bins they came from, relative to the maximum $20 \%$ completeness level.

In order to extend our sample to higher masses, we combined masses derived for our sample with those for stars from the survey by Preibisch et al. (2002). We chose to use the Preibisch et al. (2002) sample because (1) it constitutes the largest sample of spectroscopically confirmed low-mass stellar members, and (2) candidates were selected from an $I, R-I$ CMD in a manner similar to the methods employed to select our sample, thus providing a complementary data set. We specifically chose not to include the higher mass X-ray-selected sources (Preibisch \& Zinnecker 1999) due to the large difference in selection techniques. To provide the most analogous samples possible, we rederived masses for the 160 stars in the Preibisch et al. (2002) 


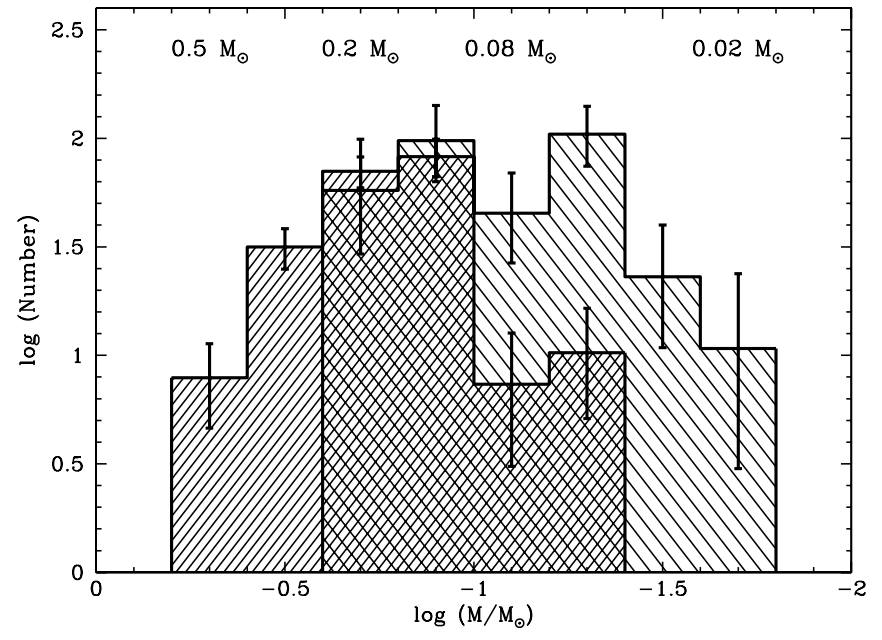

FIG. 14.- IMF for the USco association from $\sim 0.6$ to $0.02 M_{\odot}$. The light and dark hatched histograms depict the IMFs derived from the Quest-2 survey and the Preibisch et al. (2002) survey, respectively. Both IMFs have been scaled to a uniform $20 \%$ completeness, and data from the Preibisch et al. (2002) survey have been scaled to match the data presented here in the $-0.6>\log \left(M / M_{\odot}\right)>-1.0$ mass bins. All histograms are shown with $\sqrt{N}$ error bars.

survey with $\mathrm{M}$ spectral types by first converting published spectral types and 2MASS magnitudes to temperature and luminosity using the techniques described in $\S 5.1$ and then using the DM97 tracks and the same interpolation program used to generate masses for the sample of stars presented here. In combining samples from multiple sources, one must be careful to account for relative completeness. Preibisch et al. (2002) estimate spectroscopic completeness levels of $87 \%$ for stars with $0.2<M / M_{\odot}<0.8$ and $67 \%$ for stars with mass $<0.2 M_{\odot}$, whereas our corrected survey is only $20 \%$ complete across all sampled mass bins. The number of stars in the Preibisch et al. (2002) survey was thus multiplied by either ( $20 \%$ complete $/ 87 \%$ complete) for stars with $0.2<M / M_{\odot}<0.8$ or $\left(20 \%\right.$ complete $/ 67 \%$ complete) for stars with $M<0.2 M_{\odot}$. The two surveys also cover very different areas. Preibisch et al. (2002) looked at $9 \mathrm{deg}^{2}$, whereas the Quest-2 survey looked at $\sim 150 \mathrm{deg}^{2}$. However, as discussed in Paper I, SCH06, and Slesnick (2007), the Quest-2 survey coverage within this area is not complete due to several failed CCDs, gaps between the CCDs, and incomplete $r-i$ color coverage. To account for differences in area, we scaled the IMF derived from the Preibisch et al. (2002) data to match, on average, the level of the IMF derived from stars in our survey in the $-0.6>\log \left(M / M_{\odot}\right)>-1.0$ mass bins. Due to the nature of magnitude-limited surveys, the lowest mass bin (or bins, depending on bin width) of any IMF is usually incomplete relative to the rest of the derived distribution; thus, for the IMF in the combined samples, we use the values derived from the Quest-2 data to populate the $-1.0>\log \left(M / M_{\odot}\right)>$ -1.4 bins of the IMF.

Figure 14 shows IMFs for the low-mass stellar and substellar population of the USco association. In total, the combined IMF contains $\sim 377$ stars with masses as high as $\sim 0.6 M_{\odot}$ and as low as $<0.02 M_{\odot}$. The derived mass function rises with a slope of $d N / d M \propto M^{-1.13}$, peaks at $\sim 0.13 M_{\odot}$, remains high with a secondary peak at $M \sim 0.05 M_{\odot}$, and then gradually falls off through the substellar regime. Thus, we find the spectroscopic IMF for USco's low-mass population to be different from the photometric IMF derived by Lodieu et al. (2007), which had a flatter slope $d N / d M \propto M^{-0.6}$ from $0.3<M / M_{\odot}<0.01$ and peaked at $M \sim$ $0.01 M_{\odot}$. The total mass inferred from the spectroscopic IMF over the mass range $0.6-0.02 M_{\odot}$ is $\sim 48 M_{\odot}$. If we correct this number to account for the fact that the IMF shown here is only $20 \%$ complete over $\sim 40 \%$ of the entire spatial extent of the Hipparcos stars, the total mass inferred for association members less massive than $\sim 0.6 M_{\odot}$ is $\sim 600 M_{\odot}$.

\subsection{Comparisons of the Low-Mass IMF between Star-forming Regions}

Diagnostic studies of stellar populations in different locations and at varying stages of evolution are needed to explore the possibility of a universal mass function for low-mass objects. While one might expect that the IMF should vary with star formation environment, we do not yet have enough evidence to determine if such a variation exists. Aside from the work presented here, numerous studies have been carried out to characterize the lowmass stellar and substellar mass functions of other young clusters and associations in a variety of environments. Because of the intrinsic faintness of low-mass objects, most surveys are photometric. Authors then use a combination of theoretical models and statistical analysis to transform a group's CMD or luminosity function into a photometric IMF that may not accurately represent the underlying population. Spectroscopic surveys provide a more accurate assessment of the stellar membership and thus of the underlying group population.

The substellar populations of several other young star-forming regions have been studied spectroscopically using techniques similar to those presented here, and we discuss results for USco in comparison to three other regions. Luhman et al. (2003b) studied the rich cluster IC 348 in Perseus. There have been several studies of the Taurus region, namely, those by Luhman (2000, 2004), Briceño et al. (2002), and Luhman et al. (2003a), which together constitute a complete sample within a selected region. Slesnick et al. (2004) used near-infrared imaging and spectroscopic observations to constrain the low-mass IMF in the $\sim 1$ Myr old Orion Nebula Cluster (ONC). The data for IC 348 (Luhman et al. 2003b) and Taurus (Luhman 2000, 2004; Briceño et al. 2002; Luhman et al. 2003a) are published as complete across all mass ranges within the areas surveyed. Slesnick et al. (2004) employed similar techniques to those used here to correct the derived IMF to a uniform $40 \%$ completeness across all magnitude ranges. IC 348, Taurus, and the ONC are all young (age $\lesssim 3 \mathrm{Myr}$ ) and, similar to USco, have not yet had time to lose members through dynamical ejection. Therefore, if the low-mass IMF is universal, similar mass distributions should be observed in all four regions.

Our goal in this section is to assess the physical differences between stars formed in different star-forming environments. As described in $\S 5.1$, effective temperatures shown in Figure 8 are derived directly from observed spectral types using a theoretical or (in our case) empirical temperature scale. Observational evidence suggests that late-type young stars will have a similar mass across a large range of ages. For example, similar masses of $M \sim 0.03-0.09 M_{\odot}$ have been measured for M6-M7 binary or triple components in both the ONC ( $\sim 1$ Myr; Stassun et al. 2006) and AB Dor ( 75 Myr; Close et al. 2007). Thus, spectral type distributions should provide a reasonable approximation to mass distributions for young low-mass stars. We therefore begin this exercise by first comparing spectral type distributions found in different star-forming regions and thereby avoid introducing uncertainties that inherently arise when deriving stellar masses.

Spectral type distributions are shown in Figure 15. All four distributions are complete in a relative sense across spectral type bins for M-type stars. Distributions for members of the ONC and members of USco discovered with Quest-2 were corrected to a uniform completeness level in a manner similar to that described in $\S 5.3$. We have included in the IC 348 and Taurus spectral type 


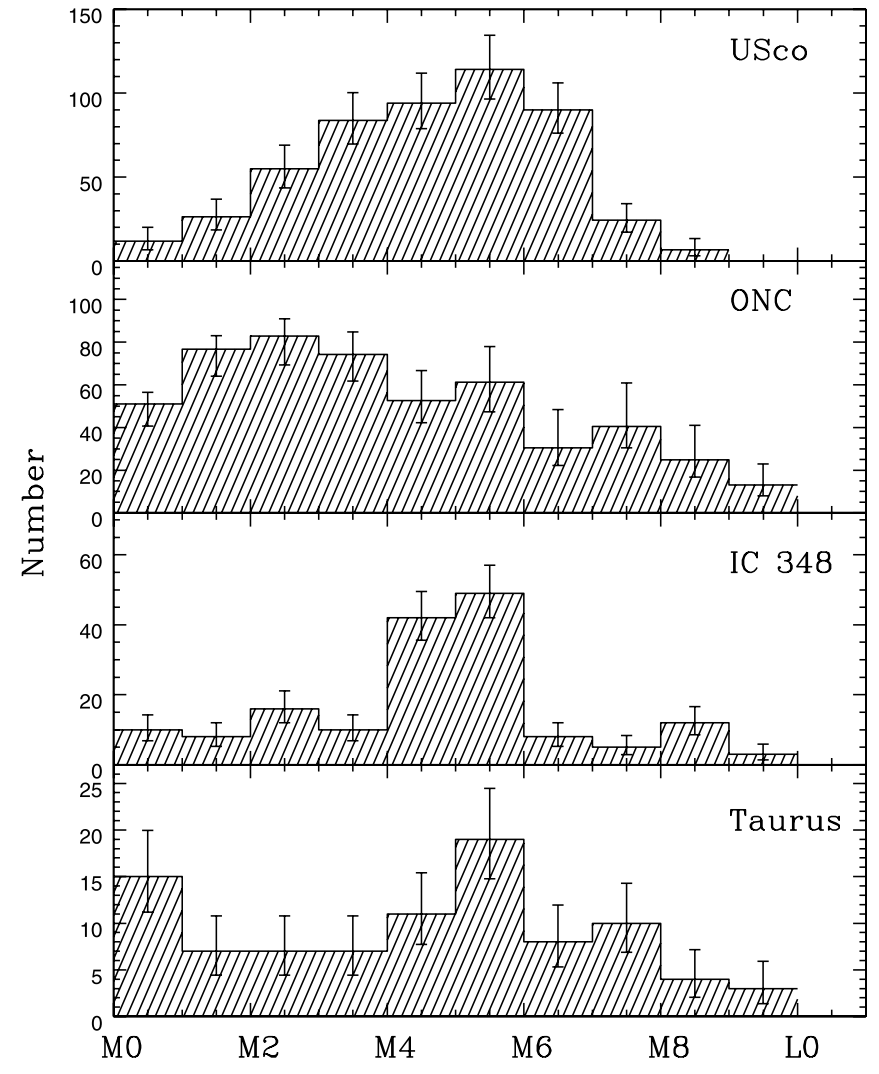

FIG. 15.-Low-mass spectral type distributions for USco, the ONC, IC 348, and Taurus. Data for the latter three regions were taken from the literature (see text).

distributions all M-type stars present in the IMF of the respective discovery paper. As can be seen, the distribution for USco is visually dissimilar to the other regions, even considering the small sample sizes and large error bars. This difference could be due to real variation in the IMF, but could also be influenced by the difference in age of a few million years ( $\sim 5$ Myr vs. $\lesssim 3 \mathrm{Myr})$ between USco and the other regions. We performed $\chi^{2}$ tests between all four distributions to determine the probabilities that the distributions could have been drawn from the same population. The tests yielded a very small probability $\left(\sim 10^{-22}\right)$ that the USco and ONC distributions could be drawn from the same population, and marginally small probabilities at the $5 \%-10 \%$ levels of USco arising from the same population as either Taurus or IC 348. However, we caution against taking results from the $\chi^{2}$ test at face value. The histograms for USco and the ONC have been scaled to account for incompleteness in magnitude and spatial area during the observations, and correctly and rigorously accounting for these scaling factors in the $\chi^{2}$ test is a nontrivial and equivocal exercise. The Taurus and IC 348 populations, however, have not been scaled and have an almost $100 \%$ chance of being drawn from the same population. While this result argues for a common spectral type distribution between these two regions amoung the lowest mass stars and brown dwarfs, substantial variation was found by Luhman et al. (2003b) for hotter, K-type stars.

We now compare directly mass distributions for the four selected star-forming regions. As discussed above and in $\S 5.1$, the mass of a star is inferred from placement on an H-R diagram using a set of theoretical mass tracks, and the exact method by which the mass is derived can vary significantly from study to study. Thus, direct comparison of the published mass data themselves requires great caution given that different mass tracks, temperature scales,

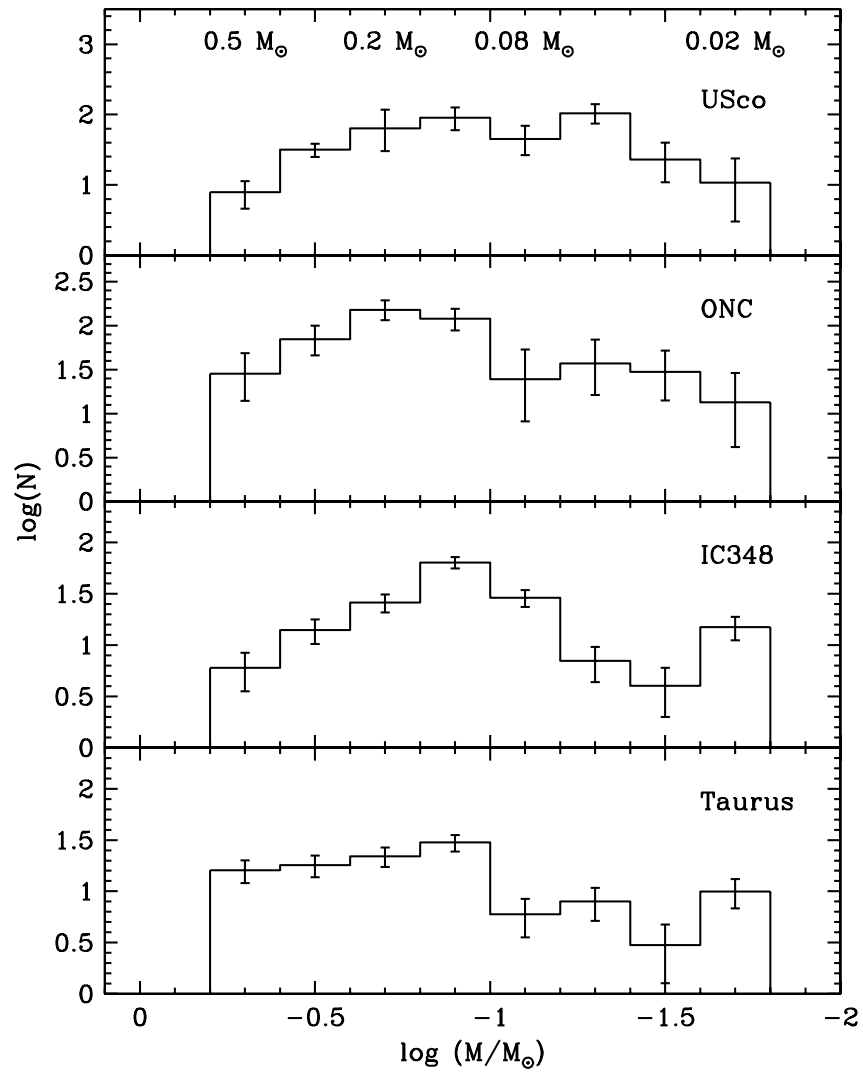

FIG. 16.- Low-mass IMFs for USco, the ONC, IC 348, and Taurus generated from DM97 mass tracks. Data for the latter three regions were taken from the literature (see text).

and interpolation methods were used for different studies. Quantitative assessment of the differences between theoretical PMS star models is beyond the scope of this work, as is analysis of differences between the numerous temperature scales employed by various authors who study young low-mass stars. We have used published spectral types and 2MASS $J, H, K_{s}$ magnitudes to derive effective temperatures, luminosities, and hence masses in a manner consistent with that used in our work in the ONC and USco. We reiterate that masses for all stars presented in this section were derived in a consistent manner; however, variations in the theoretical or empirical scales/tracks used could shift interpretation of the data.

IMFs derived from the DM97 tracks are shown in Figure 16. We find that the ONC, IC 348, and Taurus IMFs exhibit a falloff of stars beyond $\sim 0.1 M_{\odot}$. The mass distribution for USco, however, does not begin to turn over until $\sim 0.05 M_{\odot}$. Thus, similar to the spectral type distribution, the IMF derived for USco shows an overabundance of late-type, low-mass stars and suggests that the difference seen in USco's spectral type distribution did arise from a difference in its IMF, rather than from slight differences in age between USco and the other regions. Our work extends the claim by Preibisch et al. (2002) that USco may contain anomalously high numbers (compared to the field) of low-mass stars to lower masses and is suggestive that USco also contains relatively higher numbers of very low mass stars and brown dwarfs compared to other nearby star-forming regions.

USco differs from IC 348, Taurus, and the ONC in that it contains $\sim 50$ very massive, $\mathrm{O}$ - and B-type stars, almost 3 times the number found in the ONC (none are found in either Taurus or IC 348). Thus, the conclusion that USco may contain relatively more very low mass stars than other young regions may be related 
to its large number of high-mass stars. For example, Adams et al. (2006) have shown through numerical simulation that disks around lower mass stars are more susceptible to destruction (farther out than $\sim 10 \mathrm{AU}$ ) from dynamical interactions with surrounding stars of higher mass. It is possible that in a similar manner, low-mass cores moving through a giant molecular cloud are more susceptible to stripping of their accretion envelope in the presence of higher mass cores. Our results suggest that, within a star-forming region, either the presence of large numbers of very massive stars or the environmental conditions that lead to numerous massive star formation may play a large role in determining the low-mass IMF. We note that this conclusion has been drawn from results for only four different environments. Many more regions must be studied before a definitive explanation of the low-mass IMF can be derived.

\section{SUMMARY}

We have completed a large-area $g, r, i$ photometric survey of $\sim 150 \mathrm{deg}^{2}$ in and near the Upper Scorpius region of recent star formation. From these data, combined with 2MASS near-infrared magnitudes, we selected candidate new PMS association members. We present here spectral observations for a total of 243 candidates observed at either Palomar or CTIO, from which we determined $145(\sim 60 \%)$ to be bona fide new Upper Scorpius members. We measured $\mathrm{H} \alpha$ emission for all new members and determine that 15 of the 145 low-mass stars and brown dwarfs in the 5 Myr USco association are still accreting. Based on comparison of the spatial distributions of low- and high-mass association members, we find no evidence for spatial segregation in USco within the northern portion of the association.

We used photometry and spectral types to derive effective temperatures and luminosities and placed newly identified association members on an H-R diagram. These data were combined with PMS evolutionary models to derive a mass and age for each star. Using Monte Carlo simulations, we showed that, taking into account known observational errors, the observed age dispersion for the low-mass population in USco is consistent with all stars forming in a single burst $\sim 5 \mathrm{Myr}$ ago, and we place an upper limit of $\pm 3 \mathrm{Myr}$ (i.e., $60 \%$ ) on the age spread if the star formation rate has been constant in time. We also derived the first spectroscopic mass function for USco that extends into the substellar regime and compared these results to those for three other young clusters and associations.

Facilities: PO:1.2m (Quest-2), Hale (Double Spectrograph), Blanco (Hydra)

\section{APPENDIX}

Magnitudes for the Quest-2 photometric candidates spectroscopically determined to be field stars are listed in Table 3.

TABLE 3

Measured Quantities for Spectroscopically Confirmed Field Dwarfs

\begin{tabular}{|c|c|c|c|c|c|c|}
\hline $\mathrm{ID}^{\mathrm{a}}$ & $g$ & $r$ & $i$ & $J^{\mathrm{b}}$ & $H^{\mathrm{b}}$ & $K_{s}^{\mathrm{b}}$ \\
\hline SCH J15561830-24183193..... & $\ldots$ & 17.10 & 15.59 & 13.25 & 12.56 & 12.28 \\
\hline SCH J15563309-18074323....... & $\ldots$ & 20.29 & 17.67 & 14.18 & 13.61 & 13.13 \\
\hline SCH J15564167-24174525.... & $\ldots$ & 17.04 & 15.45 & 13.14 & 12.55 & 12.29 \\
\hline SCH J15572004-24462048........ & 18.89 & 17.41 & 15.76 & 13.35 & 12.74 & 12.46 \\
\hline 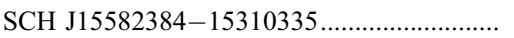 & $\ldots$ & 16.64 & 14.88 & 12.36 & 11.79 & 11.52 \\
\hline 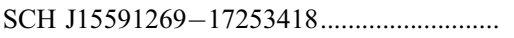 & 20.04 & 18.55 & 16.81 & 14.15 & 13.49 & 13.12 \\
\hline 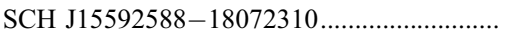 & $\ldots$ & 18.77 & 16.82 & 13.98 & 13.32 & 12.95 \\
\hline SCH J15593963-17461084.......... & 19.42 & 17.84 & 16.22 & 13.77 & 13.02 & 12.73 \\
\hline 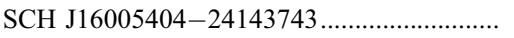 & $\ldots$ & 20.51 & 18.25 & 15.07 & 14.62 & 14.11 \\
\hline SCH J16010703-20591882 „....................... & 18.37 & 16.86 & 15.35 & 12.94 & 12.41 & 12.12 \\
\hline SCH J16021574-23435806...... & 17.28 & 15.87 & 14.43 & 12.17 & 11.48 & 11.25 \\
\hline SCH J16030530-24090255 ....... & $\ldots$ & 15.89 & 14.47 & 12.27 & 11.76 & 11.49 \\
\hline SCH J16032871-21583609...... & $\ldots$ & 19.33 & 17.54 & 14.62 & 13.96 & 13.59 \\
\hline SCH J16035236-23564424 .......... & 17.93 & 16.38 & 14.96 & 12.67 & 12.09 & 11.78 \\
\hline SCH J16070403-14352587...... & 17.85 & 16.40 & 14.67 & 12.28 & 11.73 & 11.41 \\
\hline SCH J16073811-15120357 _.......................... & $\ldots$ & 18.84 & 16.36 & 12.95 & 12.31 & 11.92 \\
\hline SCH J16080516-24334024...... & 18.17 & 16.81 & 15.26 & 13.02 & 12.44 & 12.15 \\
\hline SCH J16084199-22165277...... & 18.42 & 16.86 & 15.18 & 12.98 & 12.43 & 12.16 \\
\hline SCH J16084729-19553026_........................ & $\ldots$ & 20.08 & 18.04 & 15.05 & 14.33 & 13.94 \\
\hline SCH J16091254-21582262.... & $\ldots$ & 19.12 & 17.23 & 14.39 & 13.77 & 13.49 \\
\hline SCH J16091802-19213678....... & $\ldots$ & 19.64 & 17.51 & 14.57 & 13.88 & 13.57 \\
\hline 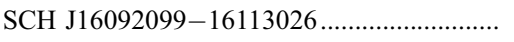 & $\ldots$ & 19.08 & 17.05 & 14.10 & 13.47 & 13.18 \\
\hline SCH J16092940-23431209...... & $\ldots$ & 19.57 & 17.54 & 14.20 & 13.57 & 13.21 \\
\hline SCH J16103948-19170814_......................... & 20.73 & 18.84 & 16.43 & 13.30 & 12.63 & 12.25 \\
\hline SCH J16104971-22223447 „....................... & 19.34 & 17.82 & 16.17 & 13.79 & 13.12 & 12.82 \\
\hline 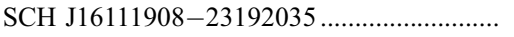 & $\ldots$ & 17.72 & 15.79 & 11.69 & 10.98 & 10.69 \\
\hline SCH J16113079-20272907 „........................ & 18.74 & 17.12 & 15.68 & 13.29 & 12.60 & 12.34 \\
\hline SCH J16113396-16330396 _....................... & $\ldots$ & 24.25 & 17.64 & 13.92 & 13.20 & 12.93 \\
\hline SCH J16122878-19590143 „........................ & $\ldots$ & 21.03 & 18.68 & 15.20 & 14.54 & 14.10 \\
\hline 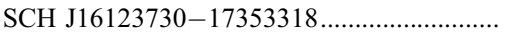 & $\ldots$ & 19.58 & 17.44 & 14.47 & 13.85 & 13.51 \\
\hline 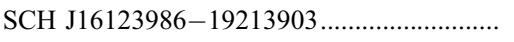 & $\ldots$ & 19.53 & 17.49 & 14.52 & 13.86 & 13.43 \\
\hline SCH J16124229-16344528 „....................... & $\ldots$ & 16.50 & 15.29 & 12.98 & 12.23 & 11.97 \\
\hline 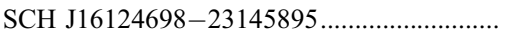 & 16.81 & 15.41 & 14.11 & 12.07 & 11.49 & 11.23 \\
\hline 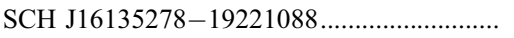 & 20.01 & 19.76 & 17.57 & 14.42 & 13.88 & 13.49 \\
\hline
\end{tabular}


TABLE 3-Continued

\begin{tabular}{|c|c|c|c|c|c|c|}
\hline $\mathrm{ID}^{\mathrm{a}}$ & $g$ & $r$ & $i$ & $J^{\mathrm{b}}$ & $H^{\mathrm{b}}$ & $K_{s}^{\mathrm{b}}$ \\
\hline SCH J16140298-19504728.. & $\ldots$ & 18.64 & 16.56 & 13.57 & 12.95 & 12.60 \\
\hline SCH J16143180-22480187 ....................... & 16.75 & 15.37 & 14.16 & 12.04 & 11.43 & 11.14 \\
\hline SCH J16144750-22460541 .......................... & 17.11 & 15.64 & 14.42 & 12.23 & 11.54 & 11.30 \\
\hline 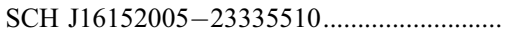 & $\ldots$ & 17.85 & 15.79 & 12.96 & 12.35 & 11.92 \\
\hline 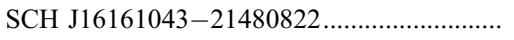 & $\ldots$ & 19.68 & 17.35 & 14.33 & 13.74 & 13.48 \\
\hline 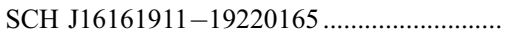 & $\ldots$ & 20.66 & 18.60 & 15.41 & 14.57 & 14.10 \\
\hline SCH J16171520-20553873 _........................ & 17.45 & 15.91 & 14.71 & 12.69 & 12.02 & 11.70 \\
\hline 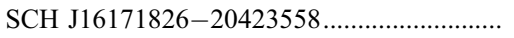 & 20.23 & 18.45 & 16.81 & 14.11 & 13.37 & 12.98 \\
\hline 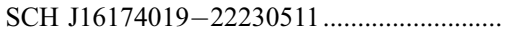 & $\ldots$ & 20.97 & 18.38 & 15.11 & 14.60 & 14.20 \\
\hline 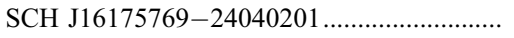 & $\ldots$ & 18.93 & 17.18 & 14.58 & 13.87 & 13.49 \\
\hline SCH J16180572-20552860 _....................... & 19.78 & 18.11 & 16.58 & 14.16 & 13.42 & 13.08 \\
\hline SCH J16192530-23470717 ........................ & $\ldots$ & 19.07 & 17.10 & 14.01 & 13.17 & 12.75 \\
\hline 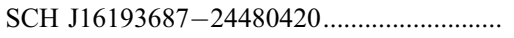 & 15.70 & 14.42 & 13.62 & 11.67 & 11.02 & 10.76 \\
\hline 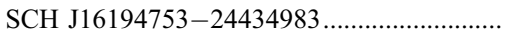 & 19.99 & 18.16 & 16.43 & 13.60 & 12.64 & 12.25 \\
\hline SCH J16194826-24291678 _....................... & 16.60 & 15.10 & 14.15 & 11.90 & 11.18 & 10.92 \\
\hline 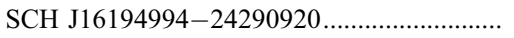 & $\ldots$ & 18.76 & 16.31 & 12.64 & 11.77 & 11.35 \\
\hline SCH J16195935-24362497..... & 15.96 & 14.72 & 13.87 & 11.88 & 11.19 & 11.02 \\
\hline SCH J16200923-20283157 ....................... & $\ldots$ & 20.63 & 18.48 & 15.09 & 14.32 & 13.89 \\
\hline 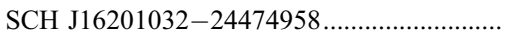 & 15.79 & 14.46 & 13.65 & 11.70 & 10.98 & 10.73 \\
\hline SCH J16202226-24460539 ......................... & $\ldots$ & 19.49 & 17.28 & 14.32 & 13.74 & 13.38 \\
\hline SCH J16202731-24282582. & $\ldots$ & 17.20 & 15.89 & 13.20 & 12.29 & 11.91 \\
\hline 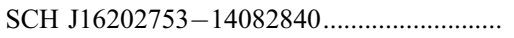 & $\ldots$ & 16.53 & 14.78 & 12.22 & 11.56 & 11.27 \\
\hline SCH J16203331-24464616......................... & $\ldots$ & 19.76 & 17.73 & 14.70 & 13.96 & 13.53 \\
\hline SCH J16205818-24380368 ....................... & 16.49 & 15.12 & 14.23 & 12.10 & 11.39 & 11.14 \\
\hline SCH J16214647-21424605 „....................... & $\ldots$ & 20.88 & 18.31 & 14.97 & 14.42 & 14.17 \\
\hline SCH J16220181-22493721 ....................... & 19.38 & 17.51 & 15.60 & 12.39 & 11.56 & 11.16 \\
\hline 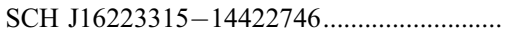 & $\ldots$ & 18.19 & 16.23 & 13.49 & 12.84 & 12.51 \\
\hline SCH J16230250-19472068_.......................... & $\ldots$ & 18.28 & 16.73 & 13.88 & 12.90 & 12.45 \\
\hline 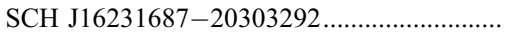 & $\ldots$ & 20.26 & 18.18 & 14.85 & 13.99 & 13.56 \\
\hline 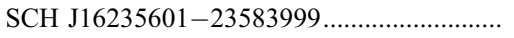 & $\ldots$ & 19.61 & 17.44 & 14.53 & 14.01 & 13.57 \\
\hline SCH J16242790-20070221 ....................... & $\ldots$ & 20.69 & 18.35 & 14.98 & 14.36 & 14.00 \\
\hline SCH J16242880-20385513 ....................... & $\ldots$ & 19.49 & 17.66 & 14.58 & 13.77 & 13.31 \\
\hline SCH J16245227-17024443 „....................... & $\ldots$ & 19.79 & 17.87 & 14.76 & 13.98 & 13.58 \\
\hline SCH J16252609-15401969 „....................... & $\ldots$ & 17.93 & 16.33 & 13.67 & 12.94 & 12.52 \\
\hline 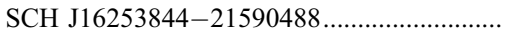 & $\ldots$ & 19.49 & 17.41 & 14.61 & 14.06 & 13.68 \\
\hline 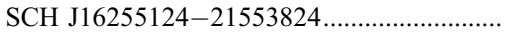 & $\ldots$ & 16.30 & 14.76 & 12.34 & 11.60 & 11.31 \\
\hline SCH J16260856-22120485 „....................... & $\ldots$ & 19.82 & 17.82 & 14.90 & 14.20 & 13.70 \\
\hline 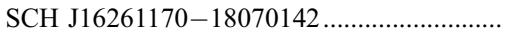 & $\ldots$ & 18.87 & 16.91 & 14.22 & 13.58 & 13.24 \\
\hline SCH J16261663-20425464 _......................... & $\ldots$ & 20.41 & 18.36 & 15.22 & 14.50 & 14.04 \\
\hline SCH J16270959-16204810 ...................... & 19.95 & 17.88 & 16.34 & 13.55 & 12.72 & 12.28 \\
\hline SCH J16280542-14053116 ........................ & $\ldots$ & 19.31 & 17.29 & 14.50 & 13.78 & 13.39 \\
\hline 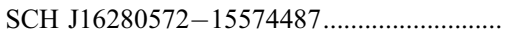 & $\ldots$ & 15.97 & 14.49 & 12.28 & 11.66 & 11.41 \\
\hline 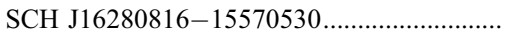 & $\ldots$ & 15.65 & 14.22 & 12.05 & 11.46 & 11.19 \\
\hline SCH J16282246-18584895 ........................ & $\ldots$ & 19.54 & 17.40 & 14.32 & 13.72 & 13.36 \\
\hline SCH J16283942-16140460 ....................... & 18.77 & 16.83 & 15.58 & 13.15 & 12.33 & 11.98 \\
\hline 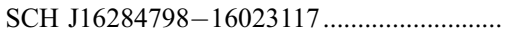 & 16.30 & 14.80 & 13.91 & 11.70 & 10.97 & 10.68 \\
\hline SCH J16290665-22464968 ........................ & $\ldots$ & 19.22 & 17.22 & 14.03 & 13.19 & 12.77 \\
\hline 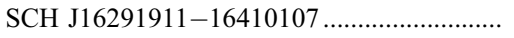 & 19.07 & 17.31 & 15.62 & 12.85 & 12.12 & 11.76 \\
\hline SCH J16293713-19172311 „...................... & $\ldots$ & 21.41 & 18.87 & 15.41 & 14.75 & 14.25 \\
\hline SCH J16300989-24243554 „....................... & $\ldots$ & 14.70 & 13.92 & 11.92 & 11.20 & 10.95 \\
\hline 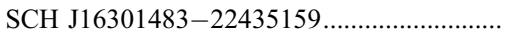 & $\ldots$ & 19.40 & 17.58 & 14.57 & 13.71 & 13.20 \\
\hline 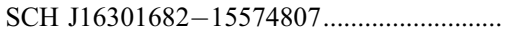 & $\ldots$ & 18.63 & 16.63 & 13.59 & 12.86 & 12.53 \\
\hline SCH J16303166-16093250 ........................ & $\ldots$ & 18.12 & 16.22 & 13.38 & 12.69 & 12.37 \\
\hline 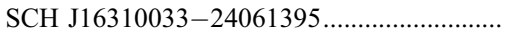 & $\ldots$ & 19.78 & 16.91 & 14.17 & 13.41 & 13.12 \\
\hline 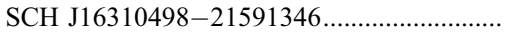 & $\ldots$ & 19.54 & 17.37 & 14.48 & 13.84 & 13.50 \\
\hline SCH J16311682-18361254 „........................ & $\ldots$ & 20.35 & 18.01 & 15.06 & 14.32 & 13.82 \\
\hline SCH J16311916-23375496 ........................ & $\ldots$ & 14.80 & 13.92 & 11.60 & 10.82 & 10.52 \\
\hline SCH J16314540-24272121 ....................... & $\ldots$ & 15.12 & 13.95 & 11.11 & 10.14 & 9.68 \\
\hline SCH J16320046-22151654 „....................... & $\ldots$ & 17.87 & 15.69 & 12.63 & 11.79 & 11.38 \\
\hline SCH J16323549-24235273 „...................... & $\ldots$ & 15.97 & 14.54 & 12.38 & 11.83 & 11.59 \\
\hline SCH J16325225-21075439 ....................... & $\ldots$ & 15.31 & 14.15 & 12.19 & 11.61 & 11.33 \\
\hline 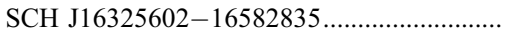 & $\ldots$ & 16.45 & 14.75 & 12.26 & 11.76 & 11.44 \\
\hline SCH J16333539-21202087 ....................... & $\ldots$ & 19.97 & 17.71 & 14.99 & 14.19 & 13.82 \\
\hline 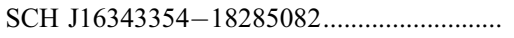 & $\ldots$ & 19.93 & 17.92 & 14.92 & 14.18 & 13.80 \\
\hline
\end{tabular}

a IDs given in $\mathrm{J} 2000.0$ coordinates.

b Near-infrared photometry taken from 2MASS. 


\section{REFERENCES}

Adams, F. C., Proszkow, E. M., Fatuzzo, M., \& Myers, P. C. 2006, ApJ, 641, 504

Ardila, D., Martín, E., \& Basri, G. 2000, AJ, 120, 479

Baltay, C., et al. 2007, PASP, 119, 1278

Baraffe, I., Chabrier, G., Allard, F., \& Hauschildt, P. H. 1998, A\&A, 337, 403

Barrado y Navascués, D., \& Martín, E. L. 2003, AJ, 126, 2997

Barsony, M., Kenyon, S. J., Lada, E. A., \& Teuben, P. J. 1997, ApJS, 112, 109

Bessell, M. S., \& Brett, J. M. 1988, PASP, 100, 1134

Blitz, L., \& Shu, F. H. 1980, ApJ, 238, 148

Briceño, C., Luhman, K. L., Hartmann, L., Stauffer, J. R., \& Kirkpatrick, J. D. 2002, ApJ, 580, 317

Carpenter, J. M. 2000, AJ, 120, 3139

Close, L. M., Thatte, N., Nielsen, E. L., Abuter, R., Clarke, F., \& Tecza, M. 2007, ApJ, 665, 736

D’Antona, F., \& Mazzitelli, I. 1997, Mem. Soc. Astron. Italiana, 68, 807 (DM97) de Bruijne, J. H. J. 1999, MNRAS, 310, 585

de Zeeuw, P. T., Hoogerwerf, R., de Bruijne, J. H. J., Brown, A. G. A., \& Blaauw, A. 1999, AJ, 117, 354

Gomez, M., Hartmann, L., Kenyon, S. J., \& Hewett, R. 1993, AJ, 105, 1927

Guieu, S., Dougados, C., Monin, J.-L., Magnier, E., \& Martín, E. L. 2006, A\&A, 446, 485

Haisch, K. E., Jr., Lada, E. A., \& Lada, C. J. 2001, ApJ, 553, L153

Hartmann, L., Ballesteros-Paredes, J., \& Bergin, E. A. 2001, ApJ, 562, 852

Hillenbrand, L. A. 2005, preprint (astro-ph/0511083)

Hillenbrand, L. A., Bauermeister, A., \& White, R. J. 2008, in ASP Conf. Ser. 384, 14th Cambridge Workshop on Cool Stars, Stellar Systems, and the Sun, ed. G. van Belle (San Francisco: ASP), 200

Hillenbrand, L. A., \& White, R. J. 2004, ApJ, 604, 741

Kraus, A. L., Ireland, M. J., Martinache, F., \& Lloyd, J. P. 2008, ApJ, 679, 762

Kraus, A. L., White, R. J., \& Hillenbrand, L. A. 2005, ApJ, 633, 452

Lada, C. J., \& Lada, E. A. 2003, ARA\&A, 41, 57

Lada, E. A., \& Lada, C. J. 1995, AJ, 109, 1682

Leggett, S. K., Allard, F., Berriman, G., Dahn, C. C., \& Hauschildt, P. H. 1996, ApJS, 104, 117

Leggett, S. K., et al. 2002, ApJ, 564, 452

Lodieu, N., Hambly, N. C., \& Jameson, R. F. 2006, MNRAS, 373, 95
Lodieu, N., Hambly, N. C., Jameson, R. F., \& Hodgkin, S. T. 2008, MNRAS, 383,1385

Lodieu, N., Hambly, N. C., Jameson, R. F., Hodgkin, S. T., Carraro, G., \& Kendall, T. R. 2007, MNRAS, 374, 372

Loinard, L., Torres, R. M., Mioduszewski, A. J., \& Rodríguez, L. F. 2008, ApJ, 675, L29

Luhman, K. L. 2000, ApJ, 544, 1044 2004, ApJ, 617, 1216

Luhman, K. L., Briceño, C., Stauffer, J. R., Hartmann, L., Barrado y Navascués, D. \& Caldwell, N. 2003a, ApJ, 590, 348

Luhman, K. L., Stauffer, J. R., Muench, A. A., Rieke, G. H., Lada, E. A., Bouvier, J., \& Lada, C. J. 2003b, ApJ, 593, 1093

Mamajek, E. E., Meyer, M. R., Hinz, P. M., Hoffmann, W. F., Cohen, M., \& Hora, J. L. 2004, ApJ, 612, 496

Martín, E. L., Delfosse, X., \& Guieu, S. 2004, AJ, 127, 449

Massey, P., Strobel, K., Barnes, J. V., \& Anderson, E. 1988, ApJ, 328, 315

Preibisch, T., Brown, A. G. A., Bridges, T., Guenther, E., \& Zinnecker, H. 2002, AJ, 124, 404

Preibisch, T., Guenther, E., \& Zinnecker, H. 2001, AJ, 121, 1040

Preibisch, T., Guenther, E., Zinnecker, H., Sterzik, M., Frink, S., \& Roeser, S. 1998, A\&A, 333, 619

Preibisch, T., \& Zinnecker, H. 1999, AJ, 117, 2381

2007, in IAU Symp. 237, Triggered Star Formation in a Turbulent ISM,

ed. B. G. Elmegreen \& J. Palous (Cambridge: Cambridge Univ. Press), 270

Rabinowitz, D., et al. 2003, BAAS, 35, 1262

Salpeter, E. E. 1955, ApJ, 121, 161

Shu, F. H., Adams, F. C., \& Lizano, S. 1987, ARA\&A, 25, 23

Skrutskie, M. F., et al. 2006, AJ, 131, 1163

Slesnick, C. L. 2007, Ph.D. thesis, Caltech

Slesnick, C. L., Carpenter, J. M., \& Hillenbrand, L. A. 2006a, AJ, 131, 3016 (Paper I)

Slesnick, C. L., Carpenter, J. M., Hillenbrand, L. A., \& Mamajek, E. E. 2006b, AJ, 132, 2665 (SCH06)

Slesnick, C. L., Hillenbrand, L. A., \& Carpenter, J. M. 2004, ApJ, 610, 1045

Stassun, K. G., Mathieu, R. D., \& Valenti, J. A. 2006, Nature, 440, 311

Wilking, B. A., Schwartz, R. D., \& Blackwell, J. H. 1987, AJ, 94, 106 